\title{
Drug Repurposing Through a Bioinformatics Pipeline Applied on Fibrotic Diseases
}

Evangelos Karatzas ${ }^{1}$, Andrea Kakouri², 3, George Kolios ${ }^{4}$, Alex Delis ${ }^{1}$ and George M. Spyrou ${ }^{2,3}$, * ${ }^{1}$ Deprt. of Informatics and Tele/coms, University of Athens, 15703, Athens, Greece, ${ }^{2}$ Deprt. of Bioinformatics, The Cyprus Inst. of Neurology and Genetics, 2370, Nicosia, Cyprus, 3The Cyprus School of Molecular Medicine, 2370, Nicosia, Cyprus, ${ }^{4}$ Deprt. of Medicine, Democritus University of Thrace, 68100, Alexandroupolis, Greece.

*Corresponding author. George M. Spyrou, Tel.: +357 22 392852; E-mail: georges@cing.ac.cy

\section{Abstract}

Subject: Fibrotic diseases cover a spectrum of systemic and organ-specific maladies that affect a large portion of the population, currently without cure. The shared characteristic these diseases feature is their uncontrollable fibrogenesis deemed responsible for the accumulated damage in the susceptible tissues. Idiopathic Pulmonary Fibrosis (IPF), an interstitial lung disease, is one of the most common and studied fibrotic diseases and still remains an active research target.

Objective: We highlight unique and common (i) genes, (ii) biological pathways and (iii) candidate repurposed drugs among nine fibrotic diseases. We bibliographically explore the resulting candidate substances for potential anti-fibrotic mode of action and focus on diseases that appear to be more similar to IPF so as to jointly examine potential treatments.

Methodology: We identify key genes for the 9 fibrotic diseases by analyzing transcriptomics datasets. We construct gene-to-gene networks for each disease and examine these networks to explore functional communities of biological pathways. We also use the most significant genes as input in Drug Repurposing (DR) tools and re-rank the resulting candidates according to their structural properties and functional relationship to each investigated disease.

Results: We identify 7 biological pathways involved in all 9 fibrotic diseases as well as pathways unique to some of these diseases. Based on our DR results, we suggest captopril and ibuprofen that both appear to slow the progression of fibrotic diseases according to existing bibliography. We also recommend nafcillin and memantine, which haven't been studied against fibrosis yet, for further wet-lab experimentation. We also observe a group of cardiomyopathy-related pathways that are exclusively highlighted for Oral Submucous Fibrosis (OSF). We suggest digoxin to be tested against OSF, since we observe cardiomyopathy-related pathways implicated in OSF and there is 
bibliographic evidence that digoxin may potentially clear myocardial fibrosis. Finally, we establish that IPF shares several involved genes, biological pathways and candidate inhibiting-drugs with Dupuytren's Disease, IgG4-related Disease, SSc and Cystic Fibrosis. We propose that treatments for these fibrotic diseases should be jointly pursued.

Keywords: Idiopathic Pulmonary Fibrosis, Drug Repurposing, Drug Re-ranking, Pathway Analysis, Disease Similarity

\section{Introduction}

Fibrotic diseases constitute a group of incurable maladies that are recognized by a fibrotic phenotype affecting various organs and tissues. Pertinent mechanisms escape the homeostatic signals and due to over-repairing, cause tissue scarring. The plethora and complexity of the underlying perturbations are responsible for the existing lack of treatments. Even though fibrotic diseases target various biological structures, they do share several underlying mechanisms (Wernig, et al., 2017; Wynn, 2007).

One of the most important fibrotic mechanisms is the extracellular matrix (ECM) deposition which is known to drive both the initiation as well as the progression of fibrogenesis (Herrera, et al., 2018). ECM is a three-dimensional network of extracellular macromolecules such as collagen, enzymes and glycoproteins, that provide structural and biochemical support to surrounding cells (Theocharis, et al., 2016). The uncontrolled accumulation of ECM macromolecules is responsible for the replacement of normal with non-functional fibrotic tissue. The transforming growth factor- $\beta$ (TGF- $\beta$ ) cytokine is a key regulator of ECM, since TGF- $\beta$ signals, and particularly SMAD proteins which are TGF- $\beta$ signal transducers, act as transcription factors that induce ECM's expression in mesenchymal cells (Verrecchia and Mauviel, 2007). Other common mechanisms among fibrotic diseases include the (i) bone morphogenic protein (BMP) signaling, (ii) overexpression of connective tissue growth factor (CTGF), (iii) Wnt/ $\beta$-catenin pathway and (iv) platelet-derived growth factor (PDGF) signaling (Rosenbloom, et al., 2017). In particular, PDGF-A-/PDGFR $\alpha$ signaling loops stimulate fibroblasts to synthesize ECM and release pro-fibrotic mediators (Bonner, 2004).

Although Idiopathic Pulmonary Fibrosis (IPF) is one of the most common and better studied fibrotic diseases, it still remains a very active research target. IPF is an interstitial lung disease (ILD), primarily involving the tissue and space around the air sacs of the lungs. IPF is of unknown etiology and due to rapid fibrotic progression, leads to death in about 3-5 years. According to (Hutchinson, et al., 2015), data from 21 countries present an incident rate of 3-9 cases per 100,000 per year for North America and Europe and lower rates for East Asia (1.2-3.8 per 100,000) and South America (0.4-1.2 per 100,000). Recent developments have led to updates in the guidelines for IPF diagnosis. In (Raghu, et al., 2018), a multidisciplinary committee provided new guidelines for IPF diagnosis by combining evidence from high-resolution computed tomography (HRCT) and histopathological patterns of 'usual interstitial pneumonia $(U I P)^{\prime}$ ', 'possible $U I P^{\prime}$ and 'indeterminate for UIP'. The recommendations strongly advise against serum biomarker (MMP7, SPD, CCL18, KL6) 
measurements as an approach to distinguish IPF from other ILDs because of the high false-positive and false-negative result rates.

In a previous work (Karatzas, et al., 2019), we analyzed fibrotic molecular and phenotypic data from the Malacards and Human Phenotype Ontology databases, for 14 fibrotic diseases in an initial effort to group fibrotic diseases and identify similarities with IPF. The diseases that we observed being more similar to IPF were Silicosis, Cystic Fibrosis (CF), Systemic Sclerosis (SSc) and IgG4-related Disease. Nevertheless, drawing conclusions regarding the similarities of the underlying mechanisms and pathogenesis among diseases still remains an open research challenge.

In this paper, we study transcriptomics datasets regarding 9 of the 14 fibrotic diseases from (Karatzas, et al., 2019) and present common and unique (i) genes, (ii) biological pathways and (iii) candidate repurposed drugs among these fibrotic diseases. We undertake this endeavor in an effort to group similar fibrotic diseases, as well as to better understand and potentially treat fibrosis. To attain our objective, we analyze gene expression datasets from microarray and next generation RNASequencing (RNA-Seq) experiments to identify over- and under-expressed genes related to each of the 9 designated diseases. We then engage respective gene lists to drive random walks on a functionally-connected network of biological pathways through our pathway analysis methodology, called PathWalks (Karatzas, et al., 2020), to identify key fibrotic pathway communities. We also use the gene lists as input into computational signature-based Drug Repurposing (DR) tools (Lussier and Chen, 2011) to help designate potential therapeutic options against fibrosis. Subsequently, we re-rank the repurposed drugs based on their drugability and functional relation to each disease (i.e., gene targets) through our CoDReS tool (Karatzas, et al., 2019). We further screen the drug candidates by prioritizing compounds that are structurally dissimilar to drugs that have already failed in clinical trials against fibrotic diseases. This is justifiable since drugs with similar structures tend to have similar mode of action (Campillos, et al., 2008). Finally, we explore the existing bibliography for evidence that further supports the potential anti-fibrotic action of our proposed drugs. We present a flowchart of our proposed analysis pipeline in Figure 1. It is in this context that we seek to establish common and unique fibrosis-related genes, pathways, and drugs in an effort to group fibrotic diseases for potential common treatments. Moreover, we identify unique characteristics among fibrotic diseases of interest so as our results can be independently verified through wet lab experimentation. 


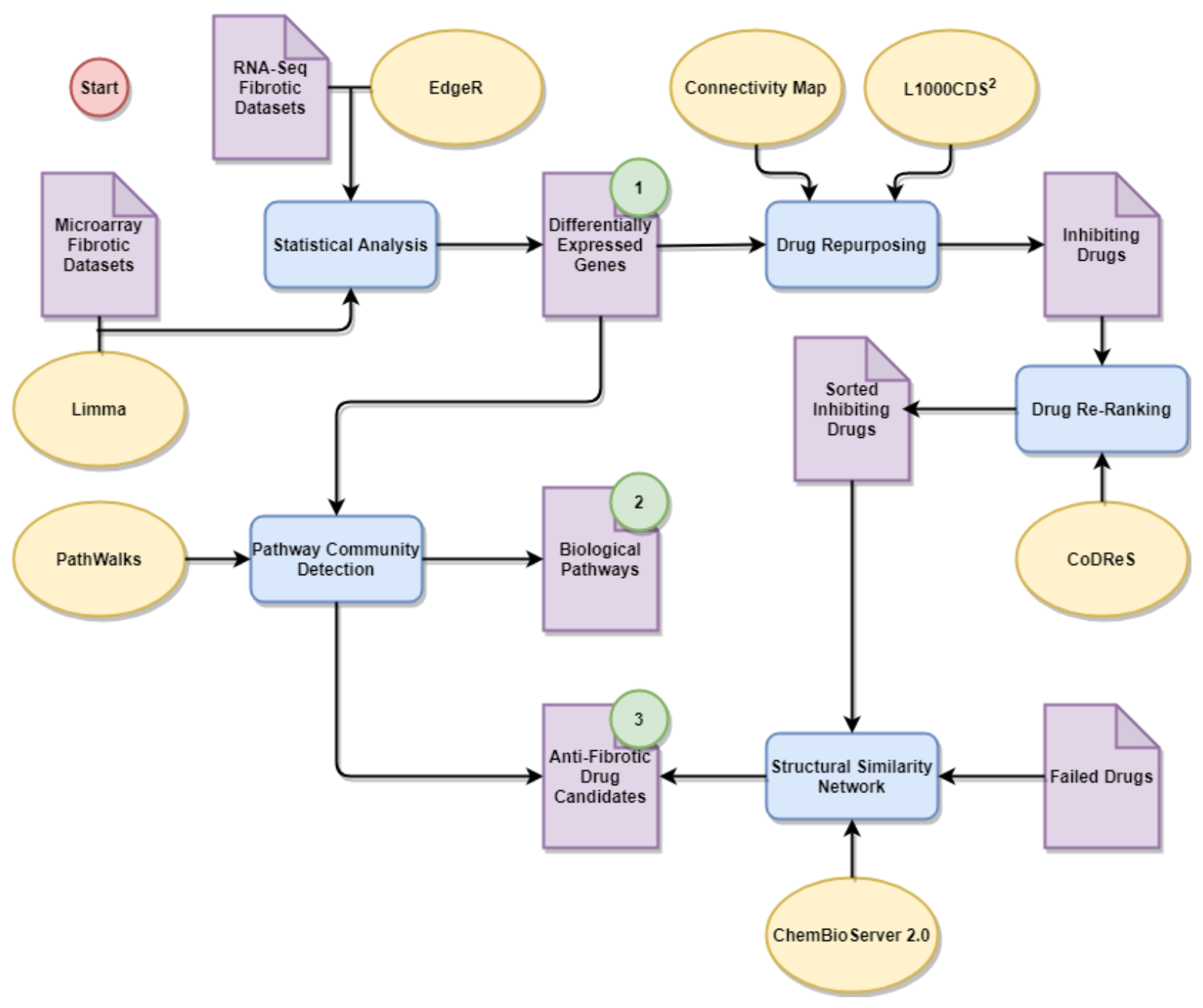

Figure 1: Flowchart of our pipeline regarding pathway analysis and drug repurposing for fibrotic diseases based on transcriptomics data. Purple pages represent data, blue rectangles represent procedures, yellow ellipses represent tools and methodologies and green circles indicate results regarding: (1) genes, (2) biological pathways and (3) inhibitingdrug candidates. We start by analyzing transcriptomics data from several tissues regarding 9 fibrotic diseases to extract key respective gene lists. We then identify common and unique perturbed biological pathways as well as potential therapeutic candidates by exploiting these gene lists.

\section{Materials and Methods}

\section{Microarray Dataset Analysis}

In the first step of the described pipeline we identify unique and common genes among fibrotic diseases by analyzing transcriptomics datasets. We then use these gene lists as input in pathway analysis and DR tools, to highlight biological pathways and candidate drugs regarding fibrosis. We initially utilize 14 microarray datasets regarding the 9 fibrotic diseases of Table 1 from Gene Expression Omnibus (GEO) (Edgar, et al., 2002). We analyze the pre-processed Series Matrix Files of the data, provided by each of these 14 experiments. We apply the normalizeQuantiles function, from the Limma package (Ritchie, et al., 2015) of the R programming language to non-normalized datasets. 
We then apply a $\log _{2}$ transformation to non- $\log _{2}$ datasets. If a dataset is already $\log _{2}$ transformed but not normalized, we remove the $\log _{2}$ transformation, then normalize and finally re-apply the $\log _{2}$ transformation. After executing the Limma statistical analysis, we use a significance cutoff-threshold ( $p$-value $<0.05)$ on the results. We remove any genes that have dual sign probes (over- and underexpressed at the same time). In the case of duplicate gene entries, we keep the result with the highest significance (i.e., lowest $p$-value score). Finally, we extract lists of the top-150 over- and top-150 underexpressed genes, based on fold change, per experiment which we then use as input in pathway analysis and DR tools.

We exclude any samples from the microarray datasets that do not correspond to the fibrotic diseases of this study. We provide some clarifications regarding these discarded samples: for the Myelofibrosis dataset GSE124281, we discard disease sample GSM3526859 since it was pooled from 2 patients and also exclude disease samples from patients with Essential Thrombocythemia. For the Oral Submucous Fibrosis (OSF) dataset GSE64216, we exclude the 2 Squamous Cell Carcinoma samples. We exclude 5 Sjogren's Syndrome samples from the IgG4-related Disease dataset GSE40568. We also exclude 7 Hepatitis B samples from the Schistosomiasis dataset GSE61376. Finally, we exclude the Non-Specific Interstitial Pneumonia (NSIP) and IPF-NSIP samples from the IPF dataset GSE110147. We also note that in the CF dataset GSE15568 patients carry the CF-specific D508 mutated CFTR-allele while in the CF dataset GSE40445 patients have a confirmed F508del homozygosity for the CFTR gene. Table 1 shows dataset details regarding diseases, sample tissues and number of samples (total, disease and control) after our exclusions. 
Table 1: Dataset information regarding the GEO microarray experiments. We analyze 4 IPF microarray datasets where all samples are derived from lung tissue. CF samples come from 2 microarray datasets from rectal mucosal epithelia and nasal epithelial cells respectively. SSc samples are derived from skin tissue. The 2 Myelofibrosis datasets contain bone marrow and bone marrow stromal cell samples respectively. The OSF samples are derived from oral buccal mucosa. The Dupuytren's Disease samples are derived from hand connective tissue. The Polycystic Kidney Disease samples are derived from renal cysts. The IgG4-related Disease samples come from labial salivary glands and finally, Schistosomiasis samples from liver tissue. Additionally, the number of samples (total, disease and control) that we experimented on is also provided for each dataset entry.

\begin{tabular}{|l|l|l|c|c|c|}
\hline \multicolumn{1}{|c|}{ Dataset ID } & \multicolumn{1}{|c|}{ Disease } & \multicolumn{1}{c|}{ Tissue } & \#Samples & \#Disease & \#Control \\
\hline GSE110147 & IPF & Lung & 33 & 22 & 11 \\
\hline GSE53845 & IPF & Lung & 48 & 40 & 8 \\
\hline GSE32537 & IPF & Lung & 207 & 167 & 50 \\
\hline GSE35145 & IPF & Lung & 8 & 4 & 4 \\
\hline GSE15568 & CF & Rectal mucosal epithelia & 29 & 16 & 13 \\
\hline GSE40445 & CF & Nasal epithelial cells & 10 & 5 & 5 \\
\hline GSE95065 & SSc & Skin & 33 & 18 & 15 \\
\hline GSE124281 & Myelofibrosis & Bone marrow & 12 & 4 & 8 \\
\hline GSE44426 & Myelofibrosis & $\begin{array}{l}\text { Bone marrow mesenchymal } \\
\text { stromal cells }\end{array}$ & 12 & 6 & 6 \\
\hline GSE64216 & OSF & Oral buccal mucosa & 6 & 4 & 2 \\
\hline GSE75152 & Dupuytren's & Hand connective tissue & 24 & 12 & 12 \\
\hline GSE7869 & Polycystic Kidney & Renal cysts & 21 & 18 & 3 \\
\hline GSE40568 & $\begin{array}{l}\text { IgG4-related } \\
\text { Disease }\end{array}$ & Labial salivary gland & 8 & 5 & 3 \\
\hline GSE61376 & Schistosomiasis & Liver & 10 & 6 & 4 \\
\hline
\end{tabular}

\section{RNA-Seq Dataset Analysis}

Complementary to the microarray dataset analysis, we use RNA-Seq datasets from GEO that are available for IPF, CF and SSc. We analyze the pre-processed Raw Count Matrix Files of the data provided by each experiment using R's EdgeR package (Robinson, et al., 2010). We perform normalization on the gene count matrices for RNA composition, by calculating the normalizing factors for the library sizes using the trimmed mean of M-values method between each pair of samples. We keep the genes with a minimum requirement of 1 count per million (CPM) across 2 or more libraries for each group (affected and control). Once we normalize the data, we then test for differential expression between patient and healthy control samples using the quasi-likelihood F-test (QLF); this is a statistical method based on negative binomial distribution that calculates the natural variation between biological replicates (Chen, et al., 2016). We then use a significance cutoff-threshold on the results $(p$-value $=0.05)$. In agreement with the microarray dataset analysis procedure, we extract lists of the top-150 over- and top-150 under-expressed genes per experiment, based on fold change.

Again, we exclude samples that do not correspond to the fibrotic diseases of this study. Regarding the GSE124548 CF dataset, we exclude the post-drug patient samples and compare pre-drug patient and healthy control samples. Similarly, for the IPF dataset GSE99621 we exclude the macroscopically 
unaffected (normal-appearing) patient samples and test for differentially expressed genes between macroscopically affected and healthy controls. Table 2 shows dataset details regarding diseases, sample tissues and number of samples (total, disease and control) after the exclusions.

Table 2: Dataset information regarding the GEO RNA-Seq experiments. We analyze 2 RNA-Seq datasets containing IPF lung samples. We also analyze 1 RNA-Seq dataset from $C F$ whole blood samples and 1 RNA-Seq dataset of $S S c$ samples from monocyte-derived macrophages. Additionally, the number of samples (total, disease and control) that we experimented on, for each dataset entry, is shown on the table.

\begin{tabular}{|l|l|l|c|c|c|}
\hline \multicolumn{1}{|c|}{ Dataset ID } & \multicolumn{1}{|c|}{ Disease } & \multicolumn{1}{c|}{ Tissue } & \#Samples & \#Disease & \#Control \\
\hline GSE99621 & IPF & Lung & 16 & 8 & 8 \\
\hline GSE134692 & IPF & Lung & 72 & 46 & 26 \\
\hline GSE124548 & CF & Whole blood & 40 & 20 & 20 \\
\hline GSE104174 & SSc & $\begin{array}{l}\text { Monocyte-derived } \\
\text { macrophages }\end{array}$ & 72 & 15 & 57 \\
\hline
\end{tabular}

\section{Pathway Community Detection}

We aggregate the key differentially expressed genes (over- and under-expressed) among experiments per disease. We utilize these gene lists in conjunction with our PathWalks methodology (Karatzas, et $a l ., 2020)$ to detect biological pathways and their functional communities that are unique or common among the 9 fibrotic diseases of this study. PathWalks is a pathway analysis and community detection methodology that guides a walker on a pathway-to-pathway network of functional connections exploiting an integrated network of genetic information that we make available.

We construct 9 gene-to-gene networks (1 per fibrotic disease) based on the extracted gene lists from the microarray and RNA-Seq datasets analysis procedure. We achieve this by using these gene lists as input in Cytoscape's (Shannon, et al., 2003) GeneMANIA plug-in (Montojo, et al., 2010). Specifically, we execute GeneMANIA with the following options: (i) 0 resultant genes and attributes (i.e., not adding enriched genes or functional categories), (ii) weight assigned based on query gene and (iii) network types based on the default selections for gene co-expression, physical interaction and pathways. We export the "normalized max weight" column of GeneMANIA's results that contains the relation weights between gene pairs. We then aggregate these weight-values among the different types of networks for the same gene pair to emphasize the relevance of the connection between the gene pair, and finally, we translate the gene symbols to their respective identifier from the KEGG database (Kanehisa, et al., 2017) to match them with the respective biological pathways they participate in.

In more detail, the GeneMANIA results present a weight for each participating type of network and a separate weight for each edge. GeneMANIA's execution algorithm consists of two parts; a label propagation function which assigns weights on the edges of the composite network and a ridge regression (regularized linear regression) function which assigns weights to the various types of networks (Mostafavi, et al., 2008). The final composite network is a weighted sum of the individual data sources. The participating networks' weights sum to $100 \%$ and reflect the relevance of each data 
source for predicting membership in the query list (Warde-Farley, et al., 2010). Edges are weighted relatively to the co-functionality between genes stated in each dataset. The final edge values are weighted sums of the respective normalized weights multiplied by the respective network's weight (Zuberi, et al., 2013). We note that each entry of GeneMANIA's "normalized max weight" column describes the normalized value for the maximum raw edge weight of a gene pair, among its various edge-entries for the same network (i.e., multiple scores from different databases for the same gene pair and network type).

Apart from the gene-to-gene networks that we implement for the PathWalks execution, a pathwayto-pathway network is also required by the algorithm. We create a network of biological pathways based on their functional relations according to the KEGG database. We assign edge scores equal to the number of common genes between each pair of pathways. We exploit the pathway-to-pathway network in all 9 executions ( 1 per disease) and finally, compare the pathway results of each disease associated to the respective genetic information networks.

The pathway nodes and their functional communities that are highlighted by PathWalks tend to favor hubs with high betweenness centrality and strength scores, as our algorithm is based on shortest paths while traversing the network of pathways. The betweenness centrality metric represents a node's participation in the total shortest paths of a network while the strength metric represents a node's sum of edge weights. We are particularly attentive to the most traversed pathways that are not necessarily highlighted due to topology but mainly through the guidance of the genetic information map. We conclude our pathway analysis by identifying pathways that are either common or uniquely perturbed in fibrotic diseases.

\section{Drug Repurposing}

We use the differentially expressed gene lists from each of the 14 microarray and 4 RNA-Seq experiments as input in 2 signature-based DR tools: (1) Connectivity Map (CMap) (Subramanian, et al., 2017) and (2) L1000CDS2 (Duan, et al., 2016). We utilize the top-150 over- and top-150 underexpressed genes from each experiment, as CMap suggests each input gene list is between 10 and 150 genes for optimal results. We need the resultant substances to inverse the input genes' (disease) signature. To achieve this in CMap, we choose the detailed list results, then select the "Compound" choice of the "Perturbagen type" subset and sort the results by ascending connectivity score. In L1000CDS² we select the "reverse" option in the "Configuration" settings on the query page since, again, we require drugs that reverse the input disease signature. We keep substances with summaryinhibition score less than -50 (-100 denotes maximum inhibition) from each CMap experiment and all 50 returned entries from L1000CDS2.

We then use our drug re-ranking tool CoDReS (Karatzas, et al., 2019) to screen the lists of drug candidates and identify the most promising inhibitors for each fibrotic disease. The tool integrates information from biological databases to calculate functional associations among input drugs and queried diseases along with their binding affinities. CoDReS also calculates the potential drugability of each drug according to the Lipinski and Veber rules (Lipinski, et al., 1997; Veber, et al., 2002). This 
tool constructs structural clusters of the input substances, based on their chemical composition, by utilizing the affinity propagation algorithm (Bodenhofer, et al., 2011) and proposes the top-ranked inhibitors per cluster for further testing against a disease. We compile an aggregated list of repurposed candidate drugs, derived from both DR tools across all experiments, for each disease and use them as input in CoDReS while selecting the respective disease for each execution.

To further screen the results, we calculate the structural similarities among the short drug lists from CoDReS and substances that have previously failed in clinical trials against fibrotic diseases. To achieve this, we construct a structural similarity network of our re-ranked drugs and the failed drugs of fibrotic diseases as found in repoDB (Brown and Patel, 2017) (last update: July 28, 2017). We use an edge cutoff-threshold of substance similarity $=0.7$. With this approach, we avoid prioritizing candidates with a lower chance of success for further experimentation, since drugs with similar chemical structures tend to have similar mode of action. We construct the structural similarity network through the ChemBioServer 2.0 web tool (Karatzas, et al., 2020) and visualize it with Cytoscape (Shannon, et al., 2003).

Subsequently, we designate the most promising anti-fibrotic candidates while focusing on IPF, by identifying the gene targets of the re-ranked drugs that participate in key implicated biological pathways. We utilize drug-target information from the DrugBank (Wishart, et al., 2018), DrugCentral (Ursu, et al., 2019) and DGIdb (Cotto, et al., 2018) databases and pathway-gene information from the KEGG pathway database (Kanehisa, et al., 2017). We finally explore the existing bibliography for antifibrotic indications concerning our proposed drug candidates to suggest the most promising for further in vitro and in vivo testing.

\section{Results}

\section{Key Fibrosis-related Genes}

In this section, we present key fibrotic genes we identify through our microarray and RNA-Seq analyses on pertinent datasets. We aggregate the top-150 over- and top-150 under-expressed gene lists among same disease experiments and observe that IPF has the most common over-expressed genes with Dupuytren's Disease (35) and the most common under-expressed genes with Myelofibrosis (28). Schistosomiasis has the least number of common gene entries with IPF (3 over- and 3 underexpressed). We identify the LCN2 gene being over-expressed in IPF, CF, Schistosomiasis and SSC and the FBLN1 gene being under-expressed in CF, Myelofibrosis, Polycystic Kidney Disease and SSc and over-expressed in IgG4-related Disease.

LCN2 encodes for the lipocalin-2 protein which is associated with neutrophil gelatinase (Kjeldsen, et al., 1993) and is known to limit bacterial growth (Flo, et al., 2004). Takahashi et al. study the LCN2 expression in (i) the skin of patients with SSc, (ii) bleomycin-treated mice, and (iii) Fli1-deficient endothelial cells. Their experiments show that $L C N 2$ is associated with dermal fibrosis in early Diffuse Cutaneous SSC cases (some of which are also diagnosed with ILD) but not with ILD markers such as diffusion lung capacity for carbon monoxide or vital capacity (Takahashi, et al., 2015). Their results 
show correlation between LCN2 and progressive skin sclerosis as well as pulmonary vascular involvement that leads to pulmonary arterial hypertension in SSc. Furthermore, (Nakagawa, et al., 2015) demonstrate increased expression of LCN2, correlated with tubulointerstitial fibrosis and tubular cell injury in patients with Chronic Kidney Disease. In another study, Kim et al. show increased LCN2 levels in urine samples of patients with Chronic Hepatitis $C$ accompanied by hepatic fibrosis (Kim, et al., 2010).

Other studies show no direct correlation between LCN2 and hepatic fibrosis. Regarding Nonalcoholic Fatty Liver Disease (NAFLD), Milner et al. observe no association between LCN2 and steatosis, lobular inflammation, ballooning or fibrosis even though the expression of LCN2 is significantly elevated in NAFLD samples compared to controls (Milner, et al., 2009). Furthermore, Borkham-Kamphorst et al. study the expression levels of LCN2 in rat models with acute and chronic liver injury. Their results show correlation of $L C N 2$ to liver damage and resulting inflammatory responses but not to the degree of liver fibrosis (Borkham-Kamphorst, et al., 2011).

Through our analysis, we observe that LCN2 is over-expressed in various fibrotic diseases and their respective targeted tissues, such as $S S c$ and the affected skin tissue. However, the correlation between the over-expression of $L C N 2$ and fibrosis is not yet clear and its role might differ among the various fibrotic diseases and affected tissues.

The FBLN1 gene encodes for the fibulin-1 protein which is an ECM and plasma glycoprotein (Argraves, et al., 1990). Chen et al. study the effects of TGF- $\beta 1$ on the regulation of FBLN1 on primary human airway smooth muscle cells from volunteers with and without Chronic Obstructive Pulmonary Disease (COPD) where small airway fibrosis occurs. They show that TGF- $\beta 1$ causes a decrease in FBLN1 mRNA and soluble FBLN1 while it increases the FBLN1 in ECM due to the sequestration of soluble FBLN1 (Chen, et al., 2013). This fact can partially justify the underexpression of FBLN1 in 4 diseases of our transcriptomics analyses (even though it was over-expressed in IgG4-related Disease), since gene expression is measured by the level of the corresponding mRNA present in a cell.

(Liu, et al., 2016) suggest that FBLN1 may be involved in the pathogenesis of not only COPD but also of asthma and IPF. Specifically, (Liu, et al., 2016) show that the deletion of FBLN1's variant FBLN1C in mouse models inhibits airway and lung remodeling in chronic asthma and lung fibrosis while also protects against COPD. The FBLN1C variant is known to increase the proliferation of lung fibroblasts in COPD and IPF patients (Ge, et al., 2015). Regarding IPF, (Jaffar, et al., 2012) identify increased soluble FBLN1 levels in the serum of patients even though there is no overexpression of FBLN1's deposition in the airway tissue. Finally, Hansen et al. study FBLN1 with regard to myocardial fibrosis. In (Hansen, et al., 2013), they suggest that, since Aortic Valve Stenosis (AVS) causes cardiac fibrosis and since they observe elevated expression of the FBLN1 gene in AVS samples against controls, the FBLN1 must be expressed as part of the fibrotic process. Even though FBLN1 is undeniably implicated in various fibrotic diseases, its expression levels in different tissues and its actual role regarding different fibrotic diseases should be further tested. 
We show the 2 common-gene participation networks (over/under-expressed) in Figure 2. Table 3 depicts the top-3 over- and top-3 under-expressed significant ( $\mathrm{p}$-value $<.05$ ) genes of each experiment based on their $\log$ fold change $(\log F C)$ values. Supplementary Tables 1 and 2 list all top common and unique genes (over- and under-expressed respectively), regarding the 9 fibrotic diseases.
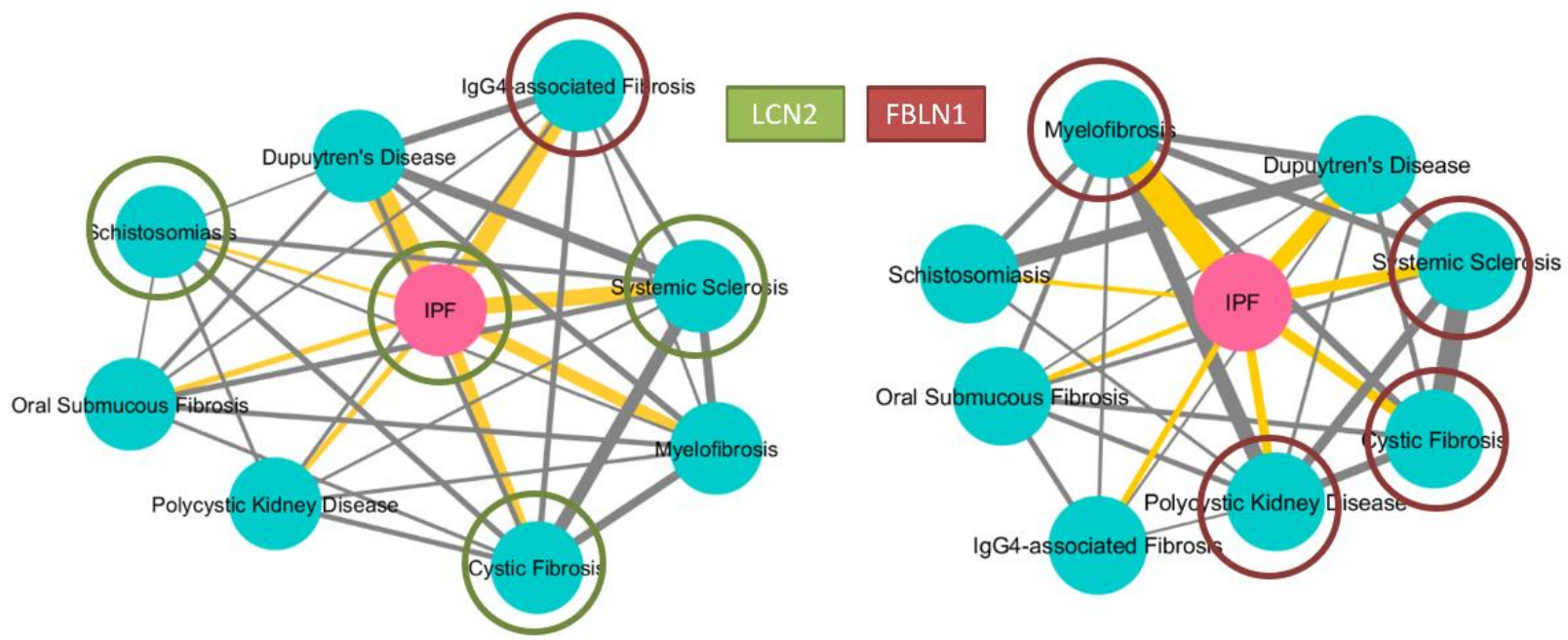

Figure 2: Common-gene participation networks. On the left network, the edge values are relative to the number of common over-expressed genes between pairs of fibrotic diseases. IPF has 35 common over-expressed genes with Dupuytren's Disease followed by 23 common with IgG4-related Disease and 20 with SSc. Our analyses indicate that the $L C N 2$ gene is over-expressed in IPF, CF, Schistosomiasis and SSc. On the right network, the edge values are relative to the number of common under-expressed genes between pairs of fibrotic diseases. IPF has 28 common under-expressed genes with Myelofibrosis followed by 16 with Dupuytren's Disease and 10 with SSc. Our analyses indicate that the FBLN1 gene is under-expressed in CF, Myelofibrosis, Polycystic Kidney Disease and SSc but over-expressed in IgG4-related Disease. Schistosomiasis has only 3 common over- and 3 common under-expressed genes with IPF.

Table 3: Top-3 over- and top-3 under-expressed genes of each experiment based on their logFC values. We acquire gene symbols from GEO, from the respective platform-translation files of each experiment.

\begin{tabular}{|c|c|c|c|c|c|c|}
\hline Dataset ID & Disease Name & $\begin{array}{l}\text { Experiment } \\
\text { Type }\end{array}$ & $\begin{array}{l}\text { Upregulated } \\
\text { Gene }\end{array}$ & $\log F C$ & $\begin{array}{c}\text { Downregulated } \\
\text { Gene }\end{array}$ & $\log \mathrm{FC}$ \\
\hline \multirow{3}{*}{ GSE110147 } & \multirow{3}{*}{$I P F$} & \multirow{3}{*}{ Microarray } & MMP1 & 4.08 & VTRNA1-1 & -5.63 \\
\hline & & & SPP1 & 3.96 & RNA5SP242 & -5.22 \\
\hline & & & BPIFB1 & 3.85 & SNORD41 & -4.33 \\
\hline \multirow{3}{*}{ GSE53845 } & \multirow{3}{*}{$I P F$} & \multirow{3}{*}{ Microarray } & MMP1 & 4.68 & PNMT & -3.97 \\
\hline & & & $U B D$ & 4.22 & IL1RL1 & -3.40 \\
\hline & & & MMP7 & 4.08 & $D E F A 3$ & -3.26 \\
\hline \multirow{3}{*}{ GSE32537 } & \multirow{3}{*}{$I P F$} & \multirow{3}{*}{ Microarray } & BPIFB1 & 2.99 & IL1R2 & -2.81 \\
\hline & & & $A S P N$ & 2.63 & MGAM & -2.17 \\
\hline & & & $M M P 7$ & 2.56 & FCN3 & -2.14 \\
\hline \multirow{3}{*}{ GSE35145 } & \multirow{3}{*}{$I P F$} & \multirow{3}{*}{ Microarray } & SFRP2 & 3.67 & IL1R2 & -4.13 \\
\hline & & & MMP7 & 3.55 & S100A12 & -3.19 \\
\hline & & & LOC649923 & 3.48 & S100A8 & -3.13 \\
\hline \multirow{3}{*}{ GSE99621 } & \multirow{3}{*}{$I P F$} & \multirow{3}{*}{ RNA-Seq } & TTR & 5.77 & CHIAP2 & -4.90 \\
\hline & & & OR51E1 & 5.75 & WFDC12 & -4.72 \\
\hline & & & GTF2H4 & 5.73 & PNMT & -4.67 \\
\hline GSE134692 & $I P F$ & RNA-Seq & ADIPOQ & 10.00 & DEFA1B & -8.99 \\
\hline
\end{tabular}




\begin{tabular}{|c|c|c|c|c|c|c|}
\hline Dataset ID & Disease Name & $\begin{array}{c}\text { Experiment } \\
\text { Type }\end{array}$ & $\begin{array}{c}\text { Upregulated } \\
\text { Gene }\end{array}$ & $\log F C$ & $\begin{array}{c}\text { Downregulated } \\
\text { Gene }\end{array}$ & $\log \mathrm{FC}$ \\
\hline \multirow{5}{*}{ GSE15568 } & & & GP1BB & 7.89 & OLFM4 & -7.50 \\
\hline & & & CST1 & 6.83 & DEFA4 & -6.95 \\
\hline & \multirow{3}{*}{$C F$} & \multirow{3}{*}{ Microarray } & $A L D O B$ & 3.08 & BRINP3 & -2.29 \\
\hline & & & ISG20 & 2.39 & SNRK & -1.70 \\
\hline & & & TNFRSF11B & 2.10 & TLR7 & -1.67 \\
\hline \multirow{3}{*}{ GSE40445 } & \multirow{3}{*}{$C F$} & \multirow{3}{*}{ Microarray } & MALAT1 & 2.60 & PROS1 & -2.39 \\
\hline & & & $L Y 6 D$ & 2.59 & CRISP2 & -2.33 \\
\hline & & & $C S F 2 R B$ & 2.25 & FABP6 & -2.32 \\
\hline \multirow{3}{*}{ GSE124548 } & \multirow{3}{*}{$C F$} & \multirow{3}{*}{ RNA-Seq } & LOC107987345 & 4.46 & RPL10P6 & -2.76 \\
\hline & & & BCORP1 & 4.45 & RPL10P9 & -1.90 \\
\hline & & & TTTY15 & 3.91 & LOC102723846 & -1.80 \\
\hline \multirow{3}{*}{ GSE95065 } & \multirow{3}{*}{$S S c$} & \multirow{3}{*}{ Microarray } & COL4A4 & 4.68 & ZBTB7C & -3.53 \\
\hline & & & SFRP4 & 3.16 & GSN & -3.22 \\
\hline & & & CXCL13 & 2.97 & $S G C A$ & -3.12 \\
\hline \multirow{3}{*}{ GSE104174 } & \multirow{3}{*}{$S S c$} & \multirow{3}{*}{ RNA-Seq } & MTND1P23 & 6.32 & LINC00221 & -3.65 \\
\hline & & & $H B A 2$ & 6.10 & DOK5 & -2.74 \\
\hline & & & CRISP3 & 4.18 & KCNJ10 & -2.16 \\
\hline \multirow{3}{*}{ GSE124281 } & \multirow{3}{*}{ Myelofibrosis } & \multirow{3}{*}{ Microarray } & IFI27 & 3.12 & LOC389342 & -1.72 \\
\hline & & & RAP1GAP & 2.29 & LOC286444 & -1.71 \\
\hline & & & IL1B & 2.03 & VCAN & -1.66 \\
\hline \multirow{3}{*}{ GSE44426 } & \multirow{3}{*}{ Myelofibrosis } & \multirow{3}{*}{ Microarray } & OXTR & 4.33 & HAND2 & -7.13 \\
\hline & & & PTPRB & 4.15 & CD70 & -6.08 \\
\hline & & & TRPC4 & 3.95 & CHI3L2 & -5.96 \\
\hline \multirow{3}{*}{ GSE64216 } & \multirow{3}{*}{ OSF } & \multirow{3}{*}{ Microarray } & $M B$ & 10.12 & KRT76 & -6.73 \\
\hline & & & MYLPF & 10.03 & LOC649923 & -6.24 \\
\hline & & & CKM & 9.81 & IGLL3 & -6.07 \\
\hline \multirow{3}{*}{ GSE75152 } & \multirow{3}{*}{ Dupuytren's } & & THBS4 & 5.94 & $L E P$ & -4.65 \\
\hline & & Microarray & LOC649366 & 4.97 & PLIN & -4.39 \\
\hline & & & TNC & 4.68 & PPP1R1A & -4.36 \\
\hline & & & UMOD & 6.84 & SFRP2 & -5.97 \\
\hline GSE7869 & Polycystic Kidney & Microarray & SLC7A13 & 6.43 & CTHRC1 & -5.62 \\
\hline & & & MIOX & 6.41 & SFRP4 & -5.49 \\
\hline & & & $A D H 1 B$ & 3.93 & FOS & -5.56 \\
\hline GSE40568 & IgG4-related & Microarray & SPP1 & 3.73 & $I G H M$ & -3.68 \\
\hline & & & $H L A-D R B 4$ & 3.23 & IFI6 & -3.57 \\
\hline & & & FAM100A & 2.85 & RPS4Y1 & -3.39 \\
\hline GSE61376 & Schistosomiasis & Microarray & $I G F B P 2$ & 2.58 & GSTM2 & -3.33 \\
\hline & & & PTMS & 2.01 & GSTM1 & -2.89 \\
\hline
\end{tabular}

\section{Identifying Fibrotic Pathway Communities}

We use the differentially expressed gene lists per disease, to drive our pathway analysis methodology. The PathWalks algorithm favors pathway-nodes with high betweenness centrality and strength scores. PathWalks, which is based on random walks and utilizes shortest paths, highlights such nodes. In Figure 3 we depict biological pathways which are more likely to appear in the PathWalks results due to the topology of the network rather than pathways with direct biological association to each fibrotic disease. Specifically, the depicted orange nodes belong to the top-5\% of 
pathways with the highest betweenness centrality. Pink nodes belong to the top-5\% of pathways with the highest degree. Red nodes belong to both groups and yellow nodes depict pathways that do not belong to any of the above 2 groups. Yellow nodes still reach the top-5\% of the PathWalks results with random pathway selection, due to their high functional connectivity edge values. Since we construct the pathway-to-pathway network based on functional connections, all colored pathways may be sensitive to biological perturbations in most use cases.

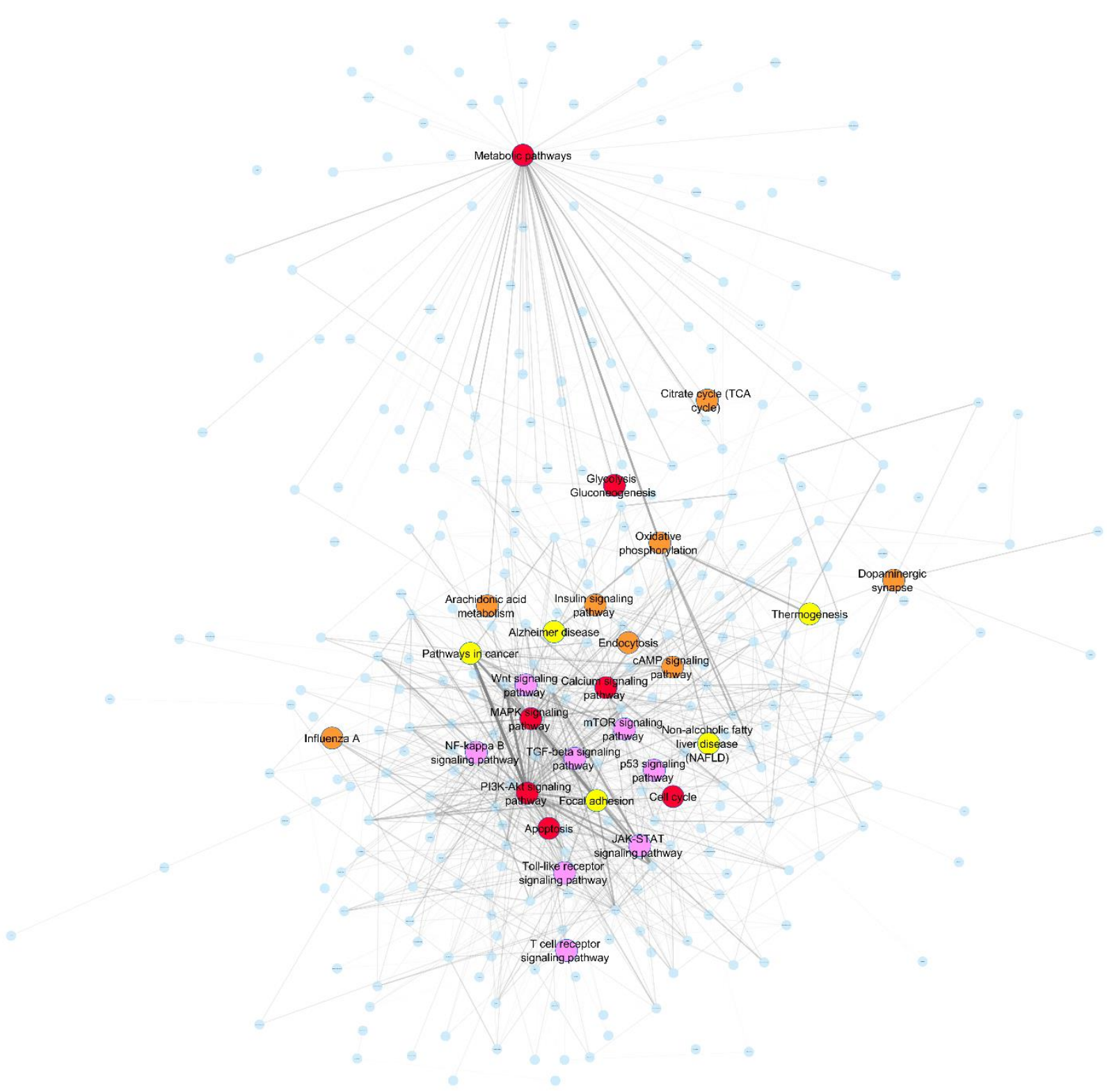

Figure 3: The base pathway-to-pathway network component that we use in the PathWalks execution. An edge indicates a functional connection between two pathways according to KEGG, and its thickness is relative to the number of their common genes. Orange nodes belong to the top- $5 \%$ of pathways with the highest betweenness centrality, pink nodes to the top-5\% with the highest degree and red nodes to both groups. Yellow nodes depict pathways that do not belong in any of these groups but still reach the top-5\% in the results of a no-map PathWalks execution (i.e., random pathway selections) due to their high functional connectivity edge values. All colored nodes are more likely to appear in the 
results due to their topological characteristics rather than due to direct relation with the genetic information networks of each disease.

We identify disease-specific as well as common implicated pathways among the 9 fibrotic maladies through the PathWalks executions. In Figure 4 and in green color, we highlight nodes that reach the top-5\% of the PathWalks results for at least 1 disease and do not belong in the topology-favored groups of Figure 3. We observe that not every topologically-favored node is highlighted by PathWalks. Therefore, we believe that even the topologically-favored nodes that are highlighted by the algorithm are not necessarily false-positive entries and should be considered for further experimentation regarding fibrosis. We then focus on the PathWalks-highlighted pathways of all 9 fibrotic diseases and draw the respective convex hulls per disease in Figure 5. We observe 7 common pathways across the 9 diseases, all of which are favored by the topology. The pathways in question are "Metabolic", "Cancer", "MAPK signaling", "PI3K-Akt signaling", "Non-alcoholic fatty liver disease", "Oxidative phosphorylation" and "Calcium signaling" pathways.

We also observe unique PathWalks-highlighted pathways in some of the fibrotic diseases. The "Cell adhesion molecules" pathway is exclusive for Myelofibrosis, the "Antigen processing and presentation" is exclusive for SSc and the "IL-17 signaling" is unique for CF. "Fat digestion and absorption", "PPAR signaling" and "Glycerolipid metabolism" pathways are unique for Dupuytren's Disease. "Influenza A", "RNA transport", "Ribosome biogenesis in eukaryotes" and "Herpes simplex virus 1 infection" pathways are unique for Schistosomiasis. Finally, the "Dilated cardiomyopathy", "Cardiac muscle contraction", "Hypertrophic cardiomyopathy", "Adrenergic signaling in cardiomyocytes" and "ECM-receptor interaction" pathways are unique for OSF.

We observe that 4 out of the 5 unique pathways of OSF are related to cardiomyopathies. A potential connection between OSF and cardiomyopathies is missing from the bibliography. However, in their review, Jiang et al. highlight drugs from various categories such as steroids, enzymes, cardiovascular drugs, antioxidants, vitamins, and microelements that seem to ameliorate (but not cure) the fibrotic effects of OSF (Jiang and $\mathrm{Hu}$, 2009). In particular, they present cardiovascular drugs that were used in other studies against OSF including (i) pentoxifylline (Rajendran, et al., 2006), (ii) buflomedil hydrochloride (Lai, et al., 1995) and (iii) nylidrin hydrochloride (Sharma, et al., 1987) as well as (iv) tea pigment (Li and Tang, 1998) all of which show temporary symptomatic relief. We suggest that a combinatorial treatment including a cardiovascular agent targeting unique highlighted cardiacrelated pathways of the OSF disease should be further pursued.

In Figure 6, we present a similarity network based on common pathway terms among the 9 fibrotic diseases. IPF does not have any exclusive pathway nodes at the top-5\% of its PathWalks results. IPF shares the most pathways with IgG4-related Disease (14/15) and the fewest with OSF (8/15). More information regarding the highlighted unique and in-common pathways among the 9 fibrotic diseases is shown in Supplementary Table 3. In Table 4, we present all pathways that were returned at the top-5\% of the PathWalks results for at least 1 experiment. We also delineate entries at the top$5 \%$ of pathways with highest betweenness centrality and degree scores as well as entries at the top- 
$5 \%$ results of a random PathWalks execution (without the use of a gene map). Here, we highlight pathways that were produced by our algorithm exclusively due to their association with the genetic information maps in green color.

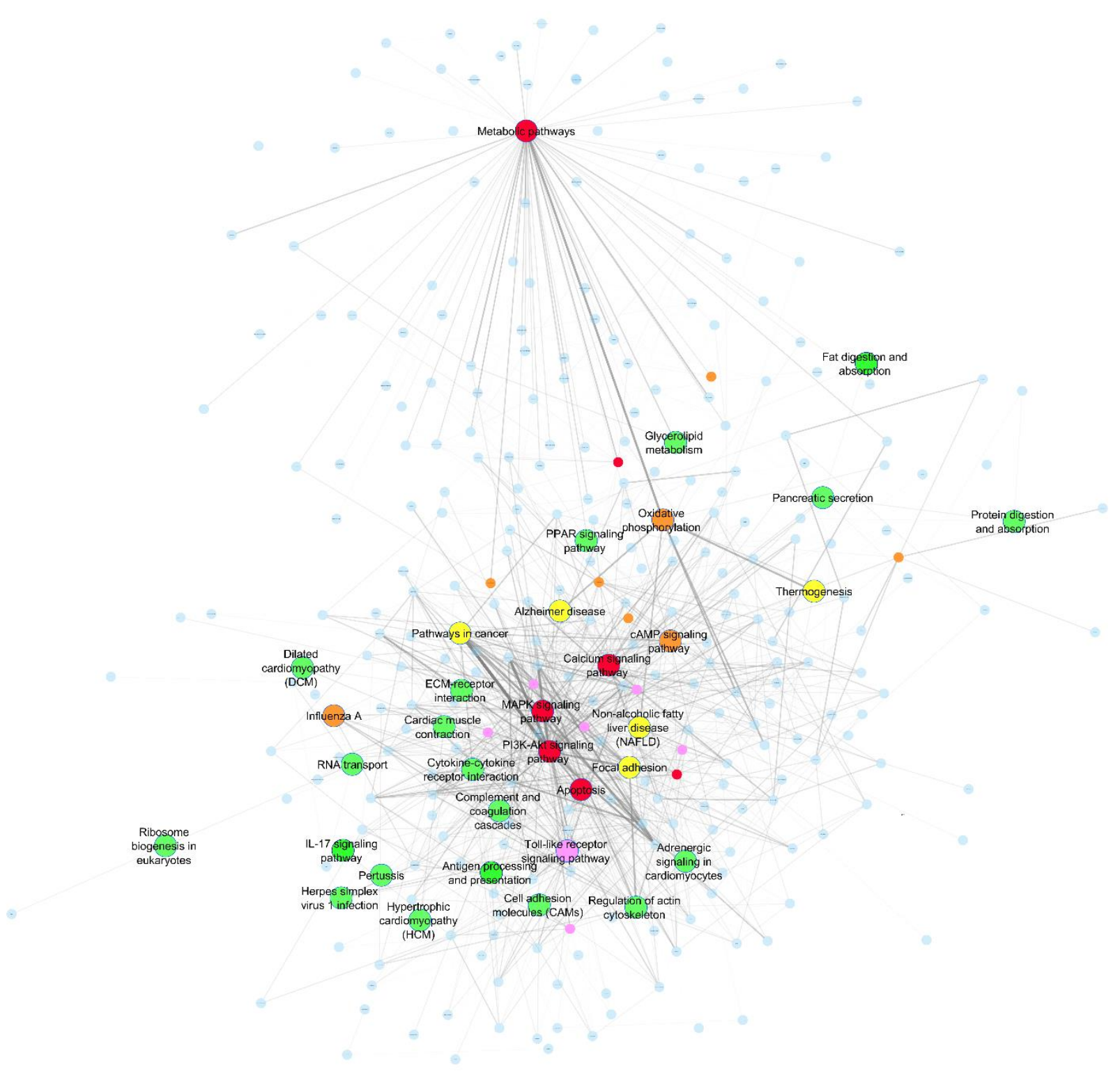

Figure 4: PathWalks results annotated on the basis of Figure 3 pathway-to-pathway. We highlight nodes that are exclusively based on the guidance of the gene maps in green color. We label all nodes that were produced in the top-5\% of any PathWalks experiment. We observe that not every topologically-favored node is highlighted by PathWalks. Hence, we believe that even the topologically-favored nodes that are highlighted by the algorithm are not necessarily false-positive entries and should be further explored regarding fibrosis. 


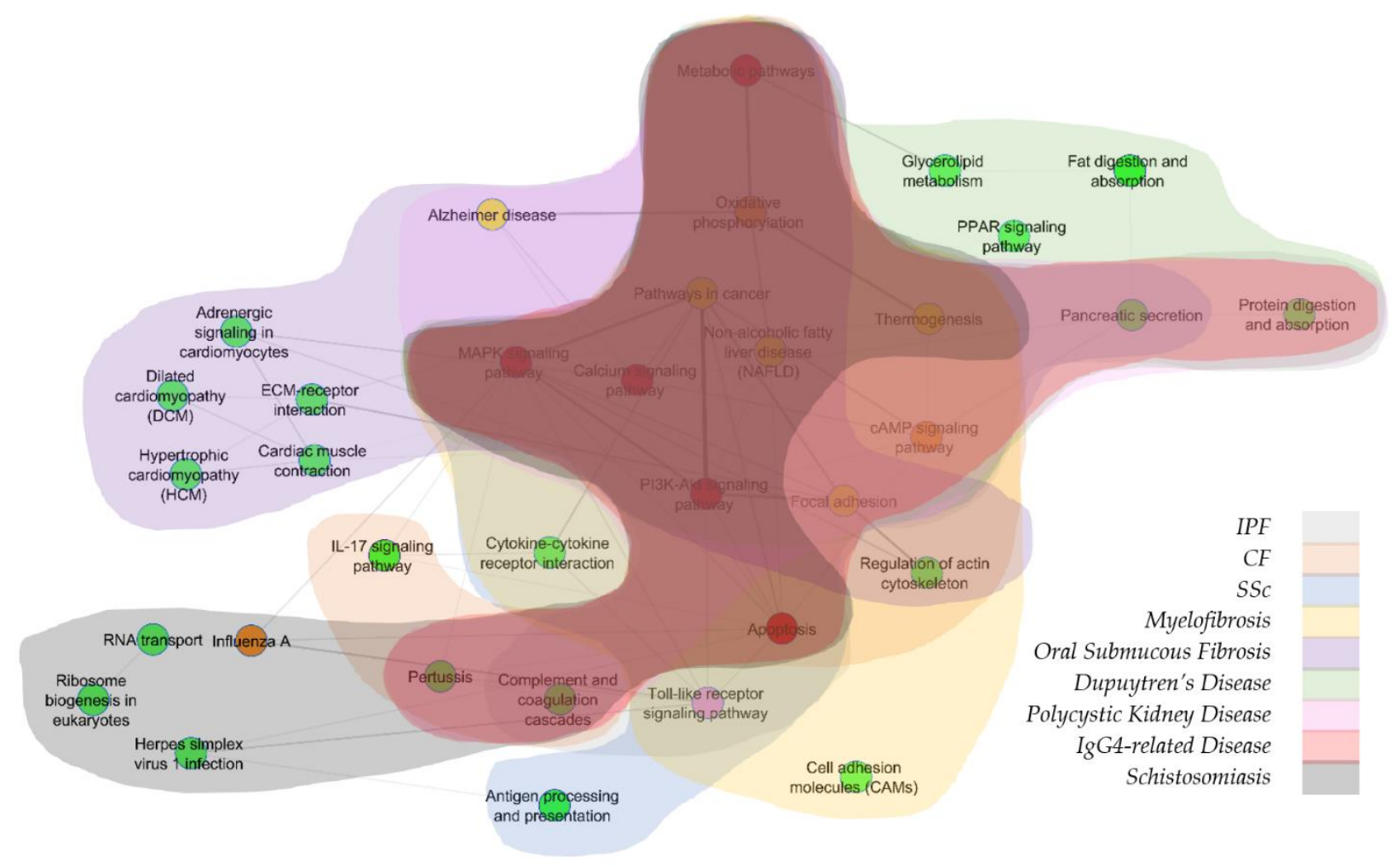

Figure 5: Convex hulls for the 9 fibrotic diseases highlighted pathways as produced by PathWalks. We observe that 7 pathways are common for all diseases including "Metabolic pathways", "Pathways in cancer", "MAPK signaling pathway", "PI3K-Akt signaling pathway", "Non-alcoholic fatty liver disease", "Oxidative phosphorylation" and "Calcium signaling pathway". We also identify unique highlighted pathways for some of the diseases. The "Cell adhesion molecules" pathway which belongs to the yellow convex hull is exclusive for Myelofibrosis. The "Antigen processing and presentation" pathway is included in the blue convex hull and is unique for SSc. The "IL-17 signaling" pathway is included in the orange convex hull and is unique for CF. "Fat digestion and absorption", "PPAR signaling" and "Glycerolipid metabolism" pathways are unique for Dupuytren's Disease and belong to the green convex hull. "Influenza A", "RNA transport", "Ribosome biogenesis in eukaryotes" and "Herpes simplex virus 1 infection" pathways belong to the dark gray convex hull and are unique for Schistosomiasis. Finally, the purple convex hull contains the "Dilated cardiomyopathy", "Cardiac muscle contraction", "Hypertrophic cardiomyopathy", "Adrenergic signaling in cardiomyocytes" and "ECM-receptor interaction" pathways which are unique for OSF. We observe that 4 out of the 5 unique pathways of OSF are related to cardiomyopathies hinting for a novel approach of potential treatments since a link between OSF and cardiomyopathies is missing from the bibliography. 


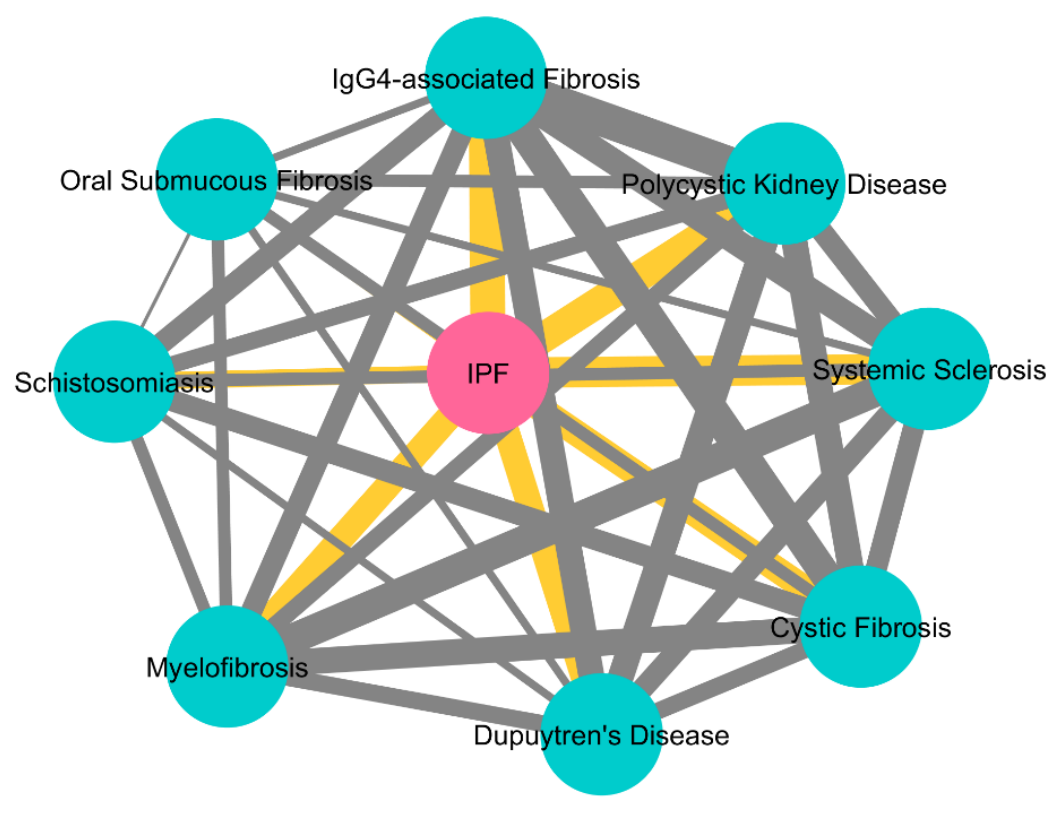

Figure 6: Similarity network among the 9 fibrotic diseases based on their common pathways as produced by the PathWalks algorithm. IPF does not have any unique pathway nodes at the top-5\% of its PathWalks results. IPF has the most common pathways with IgG4-related Disease (14/15) and the least with OSF (8/15). The edge thickness is relative to the number of common pathways between any 2 diseases. 
Table 4: Participation table of pathways at the top-5\% of betweenness centrality, degree and random PathWalks (without gene map) result nodes. The $1^{\text {st }}$ column of the table contains all pathways that reached the top-5\% in at least 1 PathWalks experiment. We provide a check mark for each pathway at the top-5\% of betweenness centrality, degree and random PathWalks lists respectively. We paint pathways that are completely highlighted by our algorithm due to their relation with each genetic information map in green.

\begin{tabular}{|c|c|c|c|}
\hline \multicolumn{4}{|c|}{ Top 5\% Pathways' Table } \\
\hline Aggregated List of Top Pathways & $\begin{array}{c}\text { Betweenness } \\
\text { Centrality }\end{array}$ & Degree & $\begin{array}{c}\text { Random } \\
\text { PathWalks }\end{array}$ \\
\hline \multicolumn{4}{|l|}{ Adrenergic signaling in cardiomyocytes } \\
\hline Alzheimer disease & & & $\checkmark$ \\
\hline \multicolumn{4}{|l|}{ Antigen processing and presentation } \\
\hline Apoptosis & $\checkmark$ & $\checkmark$ & $\checkmark$ \\
\hline Calcium signaling pathway & $\checkmark$ & $\checkmark$ & $\checkmark$ \\
\hline cAMP signaling pathway & $\checkmark$ & & $\checkmark$ \\
\hline \multicolumn{4}{|l|}{ Cardiac muscle contraction } \\
\hline \multicolumn{4}{|l|}{ Cell adhesion molecules } \\
\hline \multicolumn{4}{|l|}{ Complement and coagulation cascades } \\
\hline \multicolumn{4}{|l|}{ Cytokine-cytokine receptor interaction } \\
\hline \multicolumn{4}{|l|}{ Dilated cardiomyopathy } \\
\hline \multicolumn{4}{|l|}{ ECM-receptor interaction } \\
\hline \multicolumn{4}{|l|}{ Fat digestion and absorption } \\
\hline Focal adhesion & & & $\checkmark$ \\
\hline \multicolumn{4}{|l|}{ Glycerolipid metabolism } \\
\hline \multicolumn{4}{|l|}{ Herpes simplex virus 1 infection } \\
\hline \multicolumn{4}{|l|}{ Hypertrophic cardiomyopathy } \\
\hline \multicolumn{4}{|l|}{ IL-17 signaling pathway } \\
\hline Influenza A & $\checkmark$ & & $\checkmark$ \\
\hline MAPK signaling pathway & $\checkmark$ & $\checkmark$ & $\checkmark$ \\
\hline Metabolic pathways & $\checkmark$ & $\checkmark$ & $\checkmark$ \\
\hline Non-alcoholic fatty liver disease & & & $\checkmark$ \\
\hline Oxidative phosphorylation & $\checkmark$ & & $\checkmark$ \\
\hline \multicolumn{4}{|l|}{ Pancreatic secretion } \\
\hline Pathways in cancer & & & $\checkmark$ \\
\hline \multicolumn{4}{|l|}{ Pertussis } \\
\hline PI3K-Akt signaling pathway & $\checkmark$ & $\checkmark$ & $\checkmark$ \\
\hline \multicolumn{4}{|l|}{ PPAR signaling pathway } \\
\hline \multicolumn{4}{|l|}{ Protein digestion and absorption } \\
\hline \multicolumn{4}{|l|}{ Regulation of actin cytoskeleton } \\
\hline \multicolumn{4}{|l|}{ Ribosome biogenesis in eukaryotes } \\
\hline \multicolumn{4}{|l|}{ RNA transport } \\
\hline Thermogenesis & & & $\checkmark$ \\
\hline Toll-like receptor signaling pathway & & $\checkmark$ & $\checkmark$ \\
\hline
\end{tabular}

\section{Investigating Inhibiting Substances}

Based on the procedure outlined in the Drug Repurposing segment of our Materials and Methods section, we collect repurposed drug candidates for the 9 respective fibrotic diseases through the CMap and L1000CDS2 DR tools. We then use our CoDReS re-ranking tool to extract the highestscoring, structural-representative and inhibiting substances among the repurposed drugs of each disease. For IgG4-related Disease, we arbitrarily choose the top-5 ranked drugs from each DR tool since there is no entry for this disease in CoDReS. In Table 5, we present the re-ranked candidate drugs for each disease. 
Table 5: The re-ranked repurposed drugs for the respective fibrotic diseases. The $1^{\text {st }}$ column contains the disease names while the $2^{\text {nd }}$ column contains the names of the respective maladies in the database of CoDReS. The $3^{\text {rd }}$ column indicates the highest-ranked structural representative inhibitors returned by CoDReS and the $4^{\text {th }}$ column indicates their number. IgG4-related Disease is an exception since there is no matching entry in CoDReS. For this use case we present the top-5 ranked drugs from each DR tool (CMap and L1000CDS²).

\begin{tabular}{|c|c|c|c|}
\hline Disease Name & $\begin{array}{c}\text { CoDReS } \\
\text { Disease Name }\end{array}$ & Top-Ranked Structural Representatives & $\begin{array}{l}\text { \#Top } \\
\text { Ranked } \\
\text { Drugs }\end{array}$ \\
\hline IPF & $\begin{array}{l}\text { Idiopathic } \\
\text { Pulmonary } \\
\text { Fibrosis }\end{array}$ & $\begin{array}{l}\text { bi-2536, boldine, brazilin, brompheniramine maleate, budesonide, buparlisib, } \\
\text { butein, celecoxib, ciglitazone, cinchonine, disulfiram, entinostat, epinephrine, } \\
\text { hydrocortisone, icosapent, ipriflavone, memantine, methimazole, mk-2206, pd- } \\
\text { 0325901, propofol, propranolol, rizatriptan, scriptaid, sorafenib, sunitinib, } \\
\text { testosterone, topotecan }\end{array}$ & 28 \\
\hline$C F$ & Cystic Fibrosis & $\begin{array}{l}\text { acepromazine, apigenin, damnacanthal, delcorine, didanosine, edaravone, } \\
\text { entinostat, fasudil, gefitinib, hydrocortisone, mercaptopurine, nafcillin, } \\
\text { navitoclax, nilotinib, orantinib, oxybutynin, propofol, quipazine, sb-202190, } \\
\text { staurosporine, terbutaline, trametinib, wortmannin, y-27632, ziprasidone }\end{array}$ & 25 \\
\hline$S S c$ & $\begin{array}{l}\text { Systemic } \\
\text { Scleroderma }\end{array}$ & $\begin{array}{l}\text { erlotinib, gsk-1059615, hydrocortisone, 1-690330, orphenadrine, radicicol, sb- } \\
431542\end{array}$ & 7 \\
\hline Myelofibrosis & $\begin{array}{l}\text { Myelofibrosis due } \\
\text { to another } \\
\text { disorder }\end{array}$ & $\begin{array}{l}\text { alaproclate, anisomycin, brefeldin a, dasatinib, emetine, flurandrenolide, } \\
\text { fludroxycortide, fluticasone, l-690330, narciclasine, nortriptyline, pd-0325901, pf- } \\
\text { 477736, procyclidine, regorafenib, reversine, ruxolitinib, sulpiride, } \\
\text { tranylcypromine, trifluoperazine, xanthohumol, zalcitabine }\end{array}$ & 22 \\
\hline OSF & $\begin{array}{l}\text { Oral Submucous } \\
\text { Fibrosis }\end{array}$ & $\begin{array}{l}\text { cefotiam, cephaeline, dephostatin, digoxin, enzastaurin, decafluorobutane, } \\
\text { xanthohumol }\end{array}$ & 7 \\
\hline $\begin{array}{l}\text { Dupuytren's } \\
\text { Disease }\end{array}$ & $\begin{array}{l}\text { Dupuytren's } \\
\text { Disease }\end{array}$ & $\begin{array}{l}\text { bi-2536, dasatinib, dicycloverine, fostamatinib, ibuprofen, 1-690330, memantine, } \\
\text { pha-665752, radicicol, triamcinolone, xylazine }\end{array}$ & 11 \\
\hline $\begin{array}{l}\text { Polycystic } \\
\text { Kidney Disease }\end{array}$ & $\begin{array}{l}\text { Polycystic } \\
\text { Kidney Diseases }\end{array}$ & $\begin{array}{l}\text { bosutinib, ipsapirone, loreclezole, picotamide, selumetinib, serdemetan, } \\
\text { tretinoin, wortmannin }\end{array}$ & 8 \\
\hline $\begin{array}{l}\text { IgG4-related } \\
\text { Disease }\end{array}$ & - & $\begin{array}{l}\text { BRD-K92317137, brefeldin-a, cerulenin, dexketoprofen, fenpiverinium, } \\
\text { gestrinone, importazole, indirubin, parthenolide, rhodomyrtoxin }\end{array}$ & 10 \\
\hline Schistosomiasis & Schistosomiasis & $\begin{array}{l}\text { 1-benzylimidazole, canertinib, captopril, chloroquine, dapsone, dexamethasone, } \\
\text { genipin, ibuprofen, iodophenpropit, levocabastine, mdl-28170, memantine, } \\
\text { nadolol, parthenolide, phenytoin, propantheline, resorcinol, scriptaid, sunitinib, } \\
\text { trametinib, triamterene, vemurafenib }\end{array}$ & 22 \\
\hline
\end{tabular}

There are 2 drugs suggested by CoDReS that are common among 3 of the 9 fibrotic diseases. Hydrocortisone is highlighted as an anti-fibrotic drug candidate from the re-ranking procedure for $I P F, C F$ and SSc. Similarly, memantine is highlighted in the use cases of IPF, Dupuytren's Disease and Schistosomiasis. Hydrocortisone is a corticosteroid and its mode of action against fibrotic diseases has been studied for more than half a century now. It is mostly used for the temporal treatment of OSF. More specifically, Desa et al. report relief of symptoms in OSF patients by injecting the submucosal fibrotic areas with specific dosages of hydrocortisone depending on the stage of the fibrosis (DeSa, 1957). In a more recent study, Singh et al. describe how they have been clinically using optimal doses of a combination of hydrocortisone acetate and hyaluronidase for the medical treatment of OSF during a period of 20 years (Singh, et al., 2010).

Hydrocortisone's effect has also been studied against Pulmonary Fibrosis (PF). Xu et al. show that hydrocortisone decreases overexpressed fibrotic factors, such as TGF- $\beta 1$, pulmonary type I collagen and inducible nitric oxide synthetase to normal levels in a rat model ( $\mathrm{Xu}$, et al., 2007). Regarding $C F$, 
the study of Tepper et al. suggests that the application of intravenous hydrocortisone to the standard treatment of hospitalized infants with $C F$, produces a greater or a more sustained improvement in their lung function (Tepper, et al., 1997). In addition (Zachariæ and Zachariae, 1957) demonstrate the effects of hydrocortisone on various fibrotic skin maladies. Specifically, regarding Dupuytren's Disease, they observe that the institution of hydrocortisone acetate at a mild stage keeps the tissue contracture stationary. Regarding IgG4-related Disease, Tanabe et al. propose a combination of hydrocortisone and thyroxine which result in clinical and laboratorial improvements against the disease (Tanabe, et al., 2006). Finally, Mitani et al. study the effects of hydrocortisone in a UVirradiated mouse model. The UV irradiation causes the appearance of fibroblasts and the accumulation of collagen in the model's lower dermis. In (Mitani, et al., 1999), they establish that hydrocortisone prevents both the fibrosis of lower dermis and the accumulation of ECM components.

Memantine is a low-affinity voltage-dependent uncompetitive antagonist of the N-methyl-Daspartate receptors found in nerve cells and is used for the treatment of patients with moderate to severe Alzheimer's Disease. A direct connection of memantine and fibrotic diseases does not exist in bibliography. However, the histopathological analysis by Abbaszadeh et al. show a successful pretreatment of a heart failure rat model with memantine, that attenuates myocyte necrosis and fibrosis (Abbaszadeh, et al., 2018). Similarly, Li et al. demonstrate that memantine attenuates lung inflammation in a bleomycin-induced acute lung injury mouse model ( $\mathrm{Li}$, et al., 2015). They propose that further studies should be carried out to identify memantine's mode of action regarding both the prevention as well as the treatment of lung fibrosis.

Based on the common re-ranked drug candidates from our analysis we derive a disease similarity network in Figure 7. The edge thickness is relative to the number of common re-ranked drugs between any pair of diseases. We calculate the significance of a disease sharing re-ranked drug candidates with IPF. In this regard, we use R's hypergeometric test function $\operatorname{dhyper}(x, m, n, k)$ where (i) $x$ is the number of shared CoDReS re-ranked drug candidates between IPF and another fibrotic disease, (ii) $m$ is the cardinality of the intersection between the IPF CoDReS drugs (28) and the total, unfiltered drug entries that are produced by CMap and L1000CDS ${ }^{2}$ across all the experiments of a disease, (iii) $n$ is the number of all the unfiltered repurposed drugs for the disease, excluding the shared ones with IPF, and (iv) $k$ is the number of the CoDReS re-ranked drugs for the disease. For example, CF shares $3(x)$ CoDReS re-ranked drug candidates with IPF, where $26(m)$ out of the 28 reranked IPF drugs exist in the unfiltered outputs from the two DR tools for $C F, 2448$ (n) exist in the $C F$ unfiltered DR outputs and were not highlighted for IPF through CoDReS and $25(k)$ is the number of CoDReS-highlighted $C F$ drug candidates. Therefore, the fact that $C F$ shares 3, potentially anti-fibrotic, drug candidates (entinostat, hydrocortisone, propofol) with IPF is statistically significant ( $p$-value $\simeq$ .002). Based on this test, sharing 2 or 3 re-ranked drug candidates with IPF is a statistically significant $(p$-value < .05) similarity while sharing only 1 drug may happen due to randomness. IPF shares memantine, scriptaid and sunitinib with Schistosomiasis, bi-2536 and memantine with Dupuytren's Disease, hydrocortisone with SSc and pd-0325901 with Myelofibrosis. In Supplementary Table 4, we 
offer detailed information regarding the common and unique highlighted candidate drugs among the 9 fibrotic diseases of interest.

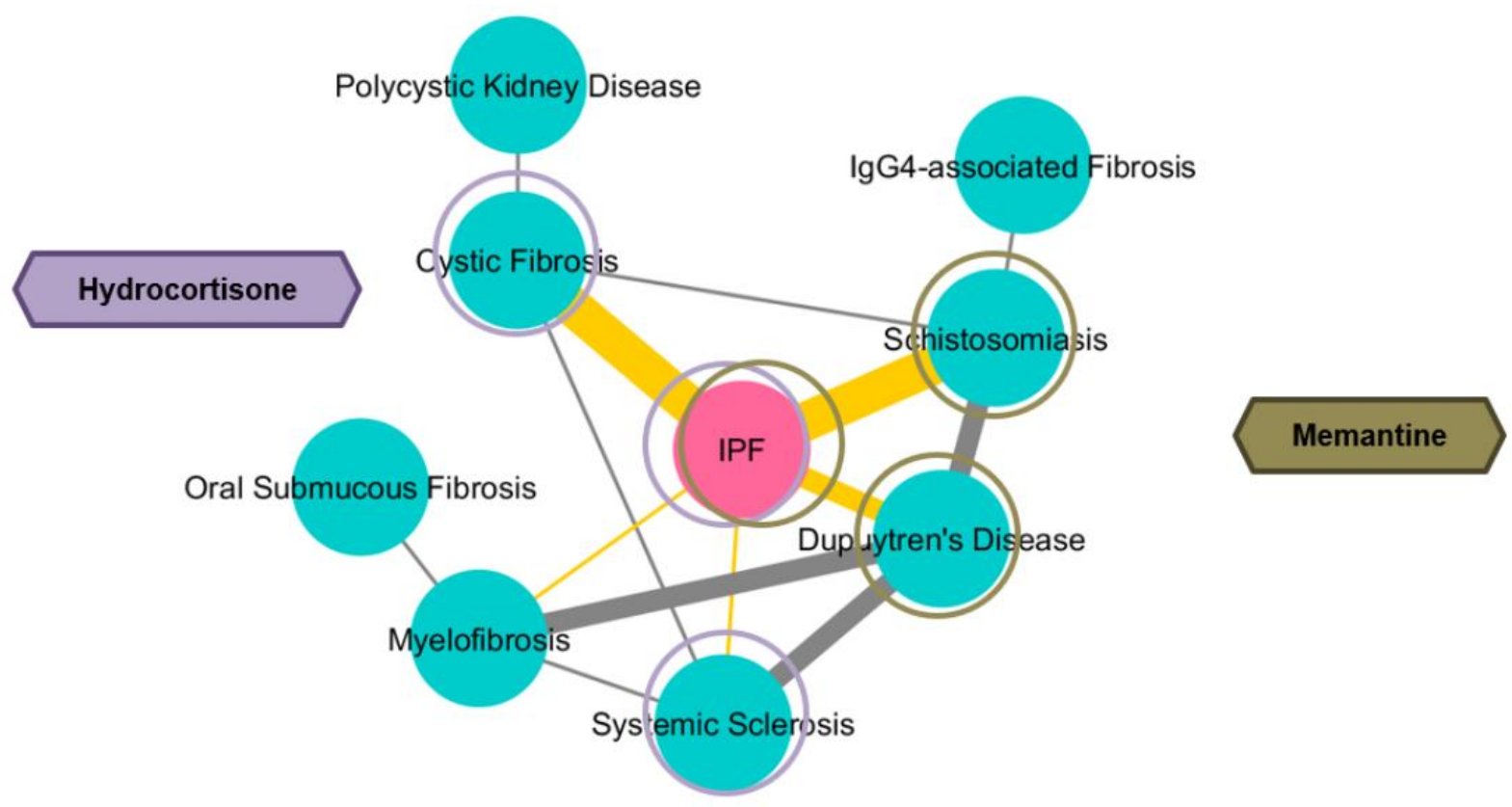

Figure 7: Disease-similarity network based on the common re-ranked drug candidates. The edge thickness is proportional to the number of common genes between pairs of diseases. IPF shares 3 drugs with CF and Schistosomiasis, 2 with Dupuytren's Disease and 1 drug with SSc and Myelofibrosis. The fact that IPF shares 2 or 3 drugs with each of CF, Schistosomiasis and Dupuytren's Disease is statistically significant based on a hypergeometric test. SSc and Myelofibrosis each share only 1 re-ranked drug with IPF, an event that might occur due to randomness. Hydrocortisone is highlighted by the re-ranking procedure for IPF, CF and SSc while memantine is highlighted in the use cases of IPF, Dupuytren's Disease and Schistosomiasis.

In an effort to further screen the repurposed and re-ranked candidate drugs, we explore structural similarities among them and identify drugs that have previously failed in clinical trials against fibrotic diseases. The repoDB database contains entries with substances and their clinical trial status against the respective diseases. The 4 different drug status types of annotation in the database are: "Approved", "Suspended", "Terminated" and "Withdrawn". We focus on drugs that have previously failed in clinical trials against any of the 9 fibrotic diseases of our study. We consider that drugs with "Suspended", "Terminated" or "Withdrawn" indications have failed in the respective clinical trial. We place detailed information regarding drug names, respective fibrotic disease and clinical trial indication combinations, as found in repoDB, in Supplementary Table 5. We note that alemtuzumab (terminated entry against SSc) does not have a chemical structure file in PubChem (Kim, et al., 2016), so we focus our analysis on the 19-rest "failed" substances.

We use PubChem-available 2-dimensional structural data files (SDF) for the aforementioned 19 failed drugs and compile an aggregated SDF file. We then compile another SDF file containing the structures of all unique $121 \mathrm{CoDReS}$ re-ranked drug candidates from this study. We use these 2 SDF 
files as input in the ChemBioServer "Attach similar-only nodes to Network" function. We use the SDF file of re-ranked drugs as a base network with an edge similarity threshold of 0.5 and the SDF file of failed drugs as a secondary list to infer structural similarity links towards the base network with an edge threshold of 0.7 . We choose the Tanimoto similarity metric since it is known to yield accurate results regarding cheminformatic similarity calculations (Bajusz, et al., 2015; Woodruff, et al., 1975). We then export the network created by ChemBioServer, remove drug nodes that are dissimilar to the failed drugs and visualize the remaining network in Cytoscape, as appears in Figure 8. With gray, we color the re-ranked drugs and with green, we color the failed substances. The edge thickness is commensurate to the structural similarity between any 2 compounds.

Figure 8 points out that hydrocortisone has high structural similarity with the "failed" drugs iloprost, mycophenolic acid and simvastatin. Hence, we suggest giving lower testing priority to hydrocortisone as well as to the rest of the substances of Figure 8 due to their high structural similarity with drugs that have already failed in clinical trials as far as fibrotic diseases are concerned. Nevertheless, we do not reject the possibility that even these potential drug choices may act as fibrotic inhibitors in conjunction with other drugs or at different doses and/or stages of fibrosis.

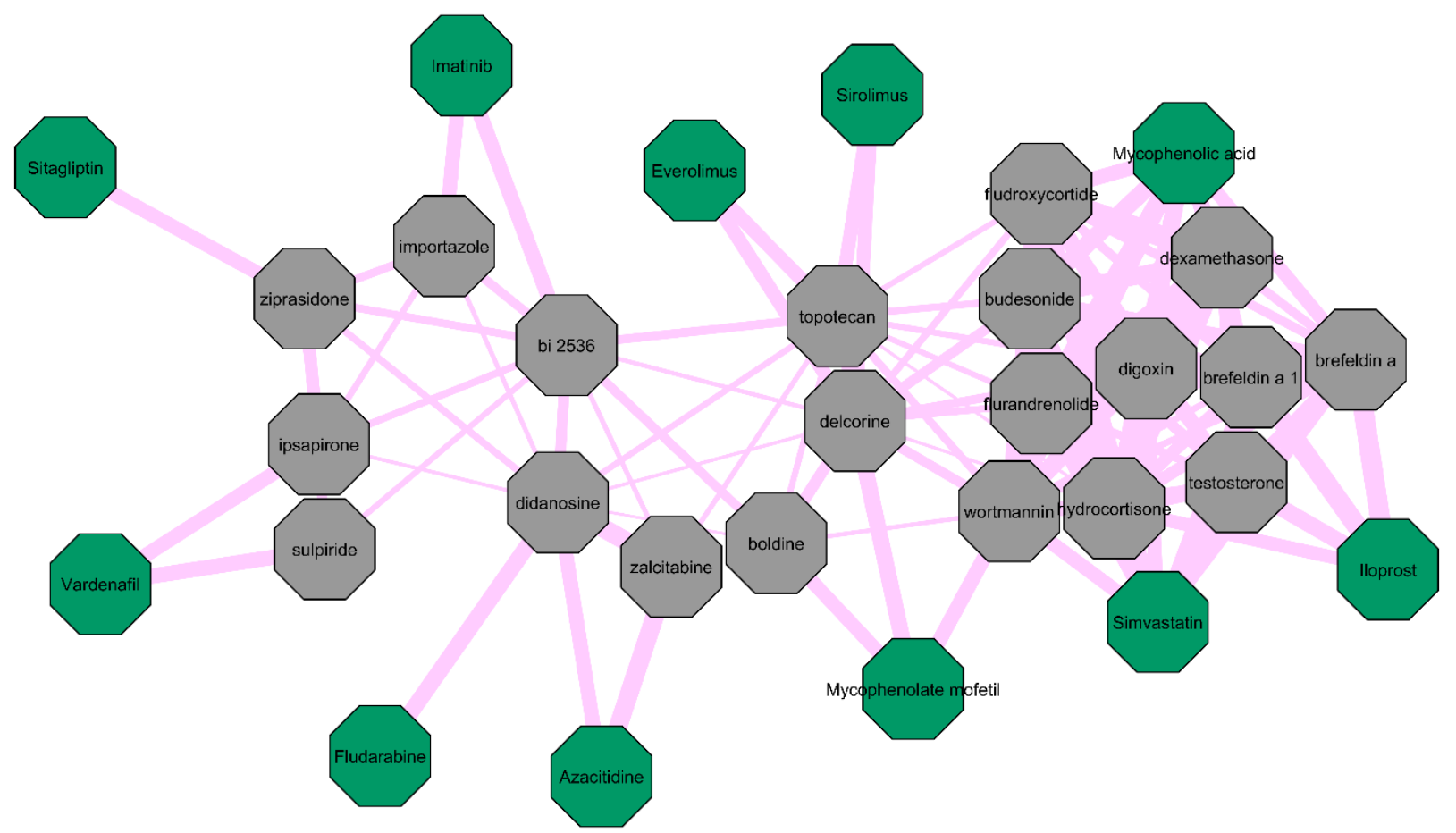

Figure 8: Structural similarity network of the CoDReS re-ranked drug candidates and drugs that have previously recorder as failed in clinical trials against fibrotic diseases. Re-ranked drugs that are dissimilar to the "failed" drugs have been removed from the network. We color the re-ranked drugs gray and the "failed" drugs green. The edge size is relative to the structural similarity between 2 compounds. Hydrocortisone, which has been re-ranked for 3 out of the 9 fibrotic diseases, has high structural similarity with iloprost, mycophenolic acid and simvastatin. We suggest giving lower testing priority to the depicted re-ranked substances due to their high similarity with already-failed drugs in clinical trials regarding fibrosis. 


\section{Highlighting Anti-fibrotic Drug Candidates}

Through our analysis pipeline, we settle for 121 total repurposed and re-ranked drug candidates with potential anti-fibrotic action. From those 121, 89 target at least 1 gene participating in at least 1 of the PathWalks highlighted pathways for the 9 fibrotic diseases. We focus on substances targeting IPF highlighted pathways that are directly associated with the gene map of IPF. There exist 3 such pathways: "Pancreatic secretion", "Protein digestion and absorption" and "Complement and coagulation cascades" colored green in Figure 9. This figure zooms into the communities of the top$5 \%$ PathWalks-highlighted pathways for IPF and their functional connections. The edge weights are proportional to the number of times they were traversed by our PathWalks algorithm. The "Pancreatic secretion" and "Protein digestion and absorption" pathways are highlighted for IPF, Dupuytren's Disease, Polycystic Kidney Disease and IgG4-related Disease. The "Pancreatic Secretion" pathway is also highlighted for SSc. The "Complement and coagulation cascades" pathway is highlighted in the cases of IPF, CF, IgG4-related Disease, Polycystic Kidney Disease and Schistosomiasis.

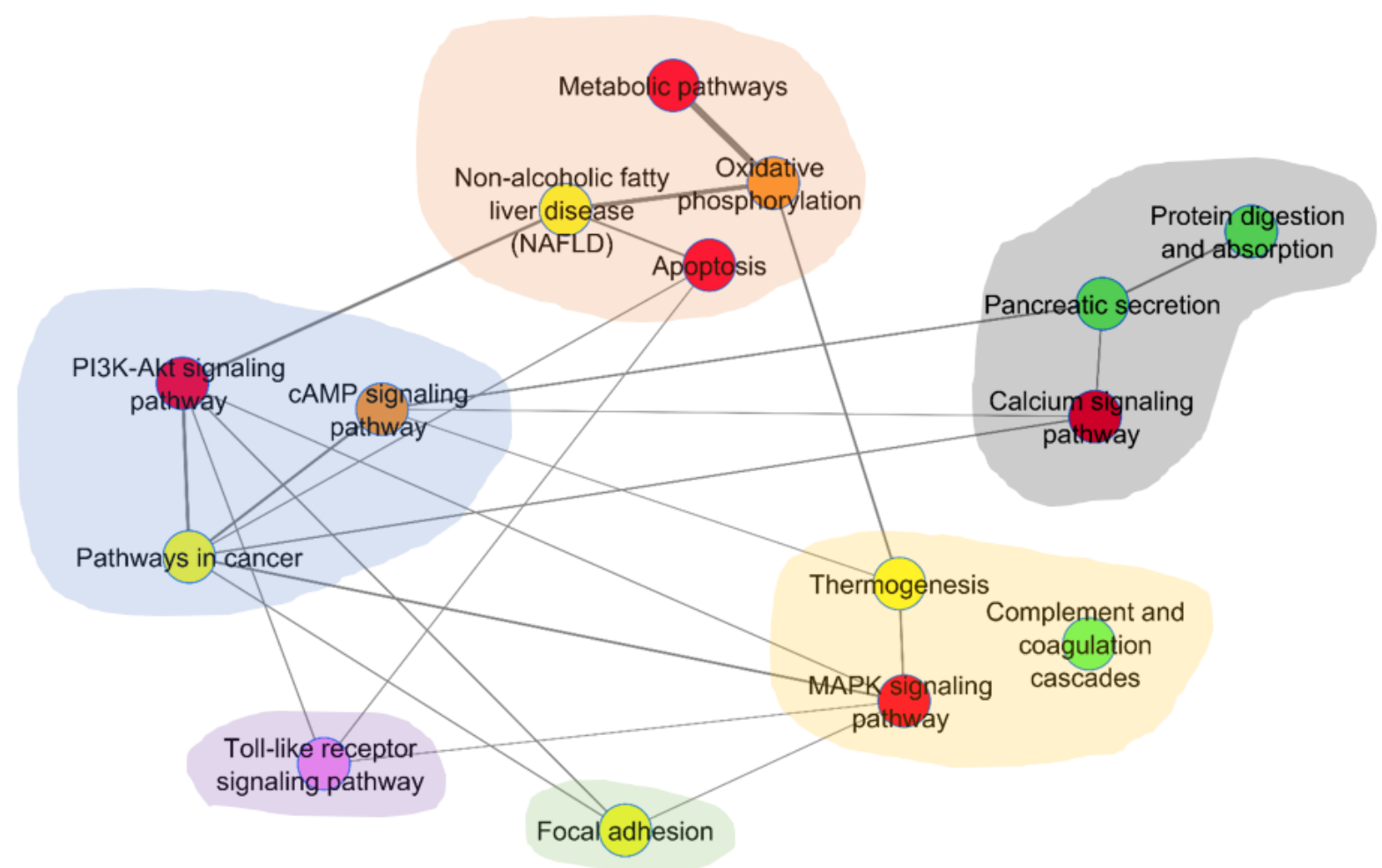

Figure 9: Pathway communities at the top-5\% results for IPF as produced by PathWalks. "Pancreatic secretion", "Protein digestion and absorption" and "Complement and coagulation cascades" are the 3 pathways that are highlighted exclusively due to their direct association with the genetic information network of IPF and are colored in green. The edge width is commensurate to the number of times an edge was traversed during the IPF PathWalks execution. The "Pancreatic secretion" and "Protein digestion and absorption" pathways are highlighted in IPF, Dupuytren's Disease, Polycystic Kidney Disease and IgG4-related Disease. The "Pancreatic Secretion" pathway is also highlighted in SSc. The "Complement and coagulation cascades" pathway is highlighted in the use cases of IPF, CF, IgG4-related Disease, Polycystic Kidney Disease and Schistosomiasis. 
There are 34 out of the 121 total re-ranked drugs that target at least 1 gene participating in the 3 "green" pathways of IPF in Figure 9. In Supplementary Table 6, we present all the combinations of re-ranked drugs, gene targets and biological pathways, where a drug has at least 1 gene target in the highlighted 'green' pathways. We bibliographically explore the 7 candidates that target at least 2 out of the 3 'green' highlighted pathways of Figure 9 for potential anti-fibrotic mode of action, namely celecoxib, digoxin, captopril, ibuprofen, staurosporine, nafcillin, wortmannin.

Celecoxib is the only candidate targeting all 3 "green" pathways of IPF. Specifically, celecoxib is an anti-inflammatory drug that we identify in the CoDReS results for IPF. Celecoxib targets the CA2 and COL18A1 genes in "Pancreatic secretion" and "Protein digestion and absorption" pathways respectively and PLAU in "Complement and coagulation cascades". We identify PLAU being overexpressed in the Dupuytren's Disease experiments. The anti-fibrotic effects of celecoxib have been widely studied, especially in rat models and mainly regarding liver fibrosis. A number of such studies (Chávez, et al., 2010; Ftahy, et al., 2013; Gao, et al., 2013; Paik, et al., 2009; Wen, et al., 2014) suggest the anti-fibrotic potential of celecoxib against hepatic fibrosis. On the other hand, contradictory studies (Hui, et al., 2006; Liu, et al., 2009) demonstrate pro-fibrotic effects of celecoxib and their results show significant exacerbated liver fibrosis and hepatocellular necrosis in celecoxibtreated rats. However, Yu et al. show that celecoxib protects against liver inflammation in early stages of fibrosis even though it has no effect on the actual fibrosis (Yu, et al., 2009). Similarly, Harris et al. observe that celecoxib treatment has no effect in the liver fibrosis of mice (Harris, et al., 2018). In a cardiac hypertrophy study, Zhang and colleagues demonstrate that celecoxib treatment prevents collagen accumulation and suppresses CTGF expression in rats (Zhang, et al., 2016). Collagen accumulation and overexpression of CTGF are established pro-fibrotic mechanisms. Nevertheless, and mostly due to contradicting studies, we suggest that other potential drug choices from our study should be prioritized instead of celecoxib for further experimentation against fibrosis.

Digoxin has been adopted for the treatment of atrial fibrillation and congestive heart failure (Hallberg, et al., 2007). We identify digoxin as a repurposed drug in the use case of OSF. As we mention in the pathway community analysis paragraph, there are 4 unique cardiomyopathy-related pathways involved in $O S F$, a finding that needs further experimental validation. Digoxin targets the "Pancreatic secretion" and "Protein digestion and absorption" through 8 genes: ATP1A1, ATP1A2, ATP1A3, ATP1A4, ATP1B1, ATP1B2, ATP1B3 and FXYD2. ATP1A2 is over-expressed in OSF and under-expressed in Dupuytren's Disease while ATP1B1 is over-expressed in Dupuytren's and FXYD2 over-expressed in Polycystic Kidney Disease. Peckham and colleagues study the effects of digoxin on nasal potential difference in $C F$ subjects with perturbed airway epithelium. Their results indicate that digoxin is unlikely to treat CF (Peckham, et al., 1995). On the other hand, Moss et al. study the effects of digoxin in $C F$ patients with heart failure complications. Their results show promise in cardiac process reversion even though it is not certain which treatment among respiratory care, vigorous antibiotic therapy or digoxin, attributed most to the survival (Moss, et al., 1975). Contrary to the previous reports, the study of Coates et al. concludes that digoxin neither increases exercise capacity 
nor improves exercising cardiac function in CF patients with moderate to severe airway obstruction (Coates, et al., 1982).

Disregarding CF, Haller et al. observe remarkable clearing of myocardial fibrosis by an affinitypurified digoxin antibody in rats with Chronic Renal Failure (Haller, et al., 2012). We observe that digoxin has high structural similarity with the "failed" drugs simvastatin and mycophenolic acid as well as hydrocortisone, as Figure 8 indicates. Nevertheless, we suggest that digoxin should be further tested specifically against OSF due to the results of our study hinting towards a link between OSF and heart diseases.

Captopril is used for the treatment of hypertension and some types of congestive heart failure (Vidt, et al., 1982) and is highlighted from the re-ranking process of our analysis in Schistosomiasis. Captopril targets the F2 and SERPINE1 genes in the "Complement and coagulation cascades" pathway and the DPP4 gene in the "Protein digestion and absorption" pathway. We observe SERPINE1 being overexpressed in Myelofibrosis and SSc but under-expressed in IPF. There are various studies highlighting captopril's anti-fibrotic mode of action in experimental rat models. Regarding lung fibrosis, GhaziKhansari's histopathological findings show that captopril helps in a paraquat-induced lung fibrosis rat model (Ghazi-Khansari, et al., 2007) while Baybutt's study shows that captopril limits fibrosis and interstitial pneumonia in a monocrotaline-induced lung fibrosis rat model (Baybutt, et al., 2007). Jalil and colleagues' study shows attenuation of both interstitial and perivascular fibrosis of myocardium in a renovascular hypertension rat model (Jalil, et al., 1991). Notable reduction of fibrosis has also been observed in rat studies regarding kidney (Cohen, et al., 1996), liver (Ramos, et al., 1994) and colon fibrosis (Wengrower, et al., 2004). We should note that there are fewer studies with contradictory results about captopril's action against fibrosis. Tuncer and others do not observe any inhibitory effects of captopril in a rat model with liver fibrosis (Tuncer, et al., 2003) while Okada's team note that captopril significantly enhances myocardial fibrosis in an isoprenaline-induced ventricular fibrosis rat model (Okada, et al., 2010). However, due to the various studies supporting captopril's anti-fibrotic action as well as the indications from our study, we suggest that captopril should be considered for further examination against fibrotic diseases and especially IPF due to a number of existing reports for its capacity to improve lung fibrosis.

Ibuprofen is an anti-inflammatory agent that is highlighted in the use cases of Dupuytren's Disease and Schistosomiasis. Ibuprofen targets the PLAT and THBD genes in the "Complement and coagulation cascades" pathway and CFTR in the "Pancreatic secretion" pathway. PLAT is overexpressed in SSc. Carlile et al. demonstrate that ibuprofen is a CF transmembrane conductance regulator (CFTR) protein corrector and suggest that ibuprofen may be suitable in a CF combination therapy (Carlile, et al., 2015). Additionally, Lindstrom et al. suggest that high doses of ibuprofen administered based on weight can treat polyposis in children with CF (Lindstrom, et al., 2007). There are 3 more studies (Konstan, et al., 1995; Konstan, et al., 2007; Lands, et al., 2007) in agreement that high doses of ibuprofen slow the progression of lung disease in CF without serious adverse effects. Therefore, ibuprofen should be further experimentally pursued in drug combinations against fibrosis. 
Staurosporine targets the PLA2G1B gene in the "Pancreatic secretion" pathway as well as COL1A2 and SLC1A1 genes in the "Protein digestion and absorption" pathway. In our analysis, we identify SLC1A1 as being over-expressed in Polycystic Kidney Disease. COL1A2 is under-expressed in Polycystic Kidney Disease but over-expressed in IPF, IgG4-related and Dupuytren's Disease. We note that the PLA2G1B gene is under-expressed in 3 out of the 6 total IPF experiments and over-expressed in 1 of them. Staurosporine is returned as a repurposed drug for CF. However, due to its non-specific selectivity on kinase-binding, staurosporine has a high chance of undesirable side effects (Karaman, et al., 2008; Tanramluk, et al., 2009). Moreover, the study of Lindroos et al. shows that staurosporine upregulates $P D G F R-\alpha$ gene expression and its protein levels in pulmonary myofibroblasts of rats (Lindroos, et al., 2001). The overexpression of PDGFR- $\alpha$ is known to induce myofibroblast hyperplasia during pulmonary fibrosis. Therefore, we consider that staurosporine is not a viable antifibrotic candidate.

We designate nafcillin, an antibiotic drug, as a repurposed and re-ranked candidate in CF. Nafcillin targets the F2RL3 gene in the "Complement and coagulation cascades" pathway and the PGA5 gene in "Protein digestion and absorption". F2RL3 is under-expressed in IPF experiments. We suggest that nafcillin's mode of action against fibrosis should be explored via cell line and model experiments since there are currently no relevant studies.

Finally, wortmannin is a phosphoinositide 3-kinase inhibitor (Powis, et al., 1994) and is highlighted by CoDReS for both CF and Polycystic Kidney Disease. Wortmannin targets the F2R and SLC1A1 genes in the "Complement and coagulation cascades" and "Protein digestion and absorption" pathways respectively. There are a few studies regarding SSc that hint at wortmannin's potential anti-fibrotic mode of action. Shi-Wen et al. report that wortmannin greatly reduces SSc patients' fibroblast ability to contract a collagen gel matrix (Shi-Wen, et al., 2004). In a later study, they add that wortmannin blocks the ability of rac1 protein to increase the expression of other profibrotic proteins (Shi-Wen, et al., 2009). Parapuram and colleagues also highlight wortmannin's capacity to reduce the expression of collagen type I, SMA and CCN2 in dermal fibroblasts from PTEN knockout mice (Parapuram, et al., 2011). The study of Zhi et al. describes that wortmannin significantly decreases the expression of collagen type I in adult rat cardiac fibroblasts (Zhi, et al., 2013). On the other hand, Wang et al. show that wortmannin cancels the effects of cannabinoid receptor type 2 agonist including improving cardiac function, decreasing fibrosis and reducing inflammatory cytokine secretion and oxidative stress in a mouse model with infarcted heart (Wang, et al., 2014). Lastly, Zhang and others also identify that wortmannin abolishes zinc's anti-fibrotic capacity in a rat kidney tubular epithelial cell line (Zhang, et al., 2016). In Figure 8, we observe that wortmannin has high structural similarity with the "failed" drugs simvastatin, mycophenolic acid and mycophenolate mofetil, as well as with the already discussed repurposed drugs of our study hydrocortisone and digoxin. Due to the contradictory results from the bibliography and wortmannin's structural similarity with already "failed" drugs against fibrosis, we do not suggest prioritizing wortmannin in future anti-fibrotic experiments. 


\section{Discussion}

In this study, we identify genes, biological pathways and potential inhibiting drugs that are associated with fibrotic diseases. Through our PathWalks methodology we identify 7 common biological pathways that are associated with all 9 fibrotic diseases investigated in this paper. These include "Metabolic", "Cancer”, "MAPK signaling”, "PI3K-Akt signaling”, "Non-alcoholic fatty liver disease", "Oxidative phosphorylation" and "Calcium signaling" pathways. We do not identify any exclusive fibrotic mechanisms for IPF but each of the Myelofibrosis, CF, SSc, Dupuytren's Disease, Schistosomiasis and OSF diseases have unique pathway entries that should be further explored as to their contribution in the pathogenesis of these diseases. We observe that the PathWalks results hint evidence for a potential common mechanism between OSF and cardiomyopathies. Therefore, we suggest that combinatorial regimens against OSF including cardiovascular agents should be looked at.

We use 2 DR tools (CMap and L1000CDS²) and the drug re-ranking tool (CoDReS) to identify and propose drug candidates with potential anti-fibrotic mode of action. We observe that hydrocortisone and memantine are returned in 3 out of the 9 fibrotic diseases of this study. Memantine's action has not been studied against fibrotic diseases but, as mentioned in our respective Results section, there are hints for its potentially beneficial anti-fibrotic action (Abbaszadeh, et al., 2018; Li, et al., 2015). As far as hydrocortisone is concerned, low doses have shown to attenuate fibrosis especially in the early stages (DeSa, 1957; Xu, et al., 2007). However, we note that hydrocortisone has high structural similarity with drugs that have previously failed in clinical trials against fibrosis. Even though we suggest prioritizing drugs that are dissimilar to "failed" ones, we do not reject the possibility that even these candidates may be effective in combination with other drugs or at different dosages and/or stages of fibrosis.

In our effort, we have also examined the repurposed drugs of this study that appear to be the most promising as anti-fibrotic choices. We select substances that target at least 2 out of the 3 PathWalks highlighted biological pathways of IPF and are directly associated with the respective gene map. We explore bibliographic evidence for pro- or anti-fibrotic mode of action regarding these drug candidates. Combining the results of our analysis and the bibliography, we suggest that captopril and ibuprofen should be prioritized for future anti-fibrotic experiments. We also suggest that nafcillin, which hasn't yet been studied against fibrosis, should be considered for in vitro and in vivo studies. Finally, digoxin should also be further explored, specifically regarding OSF since, as stated in the Results section, it appears to clear myocardial fibrosis and OSF shares multiple biological pathways related to myocardial diseases.

We observe the LCN2 gene being differentially over-expressed in 4 out of the 9 studied fibrotic diseases. Bibliographic evidence shows correlation between LCN2 and fibrosis in SSc, Chronic Hepatitis $C$ and Chronic Kidney Disease. However, in other diseases such as NAFLD and cases of liver injury, LCN2 cannot be correlated to the observed fibrosis. More experimentation is needed to better understand the role of LCN2 in fibrotic implications. We also identify FBLN1 being under-expressed 
in 4 out of the 9 fibrotic diseases and over-expressed in another. According to bibliography, even though FBLN1 mRNA levels may decrease in COPD where small airway fibrosis occurs, on the protein level it is accumulated in the ECM. FBLN1 levels are increased in serum and bronchoalveolar lavage fluid of asthma patients (Lau, et al., 2010) and in the plasma and lung tissue of IPF patients (Jaffar, et al., 2014). Based on our results, we observe an association between FBLN1 and fibrotic diseases; this is also corroborated in existing bibliography (Ge, et al., 2015; Hansen, et al., 2013; Jaffar, et al., 2012) regarding specific tissues (e.g., lung, myocardium). We suggest that further proteomics analyses should be performed to measure the quantity of the fibulin-1 translated protein in the related fibrotic tissues and its potential involvement in fibrosis.

Our work establishes gene, pathway and candidate drug similarities among IPF and the rest of fibrotic diseases. Specifically, IPF shares several terms with Dupuytren's Disease having 35 common over-expressed and 16 common under-expressed genes, 2 common key pathways with direct association to the respective genetic information maps and 2 common identified drug candidates. IPF and Myelofibrosis share 28 under-expressed genes. IPF and IgG4-related Disease share 23 overexpressed genes and 3 key pathways. Finally, IPF shares 20 over-expressed genes and 1 key pathway with SSc and 3 drug candidates and 1 key pathway with CF. Our conjecture is that common treatments for IPF and the aforementioned diseases, especially Dupuytren's Disease, should be pursued.

We provide all derived results for brevity in Supplementary Material. Supplementary Tables 1 and 2 present the top over- and under-expressed genes per disease. In Supplementary Table 3, we depict the top-5\% ranked pathways from the PathWalks algorithm and in Supplementary Table 4, we show the CoDReS re-ranked drugs that we identify for each fibrotic disease. Supplementary Table 5 presents the indications for the "failed" drugs of repoDB regarding clinical trials against fibrotic diseases. Finally, Supplementary Table 6 links drugs to their gene targets and the respective highlighted pathways. We stipulate that results on the gene, pathway and drug levels from this study will be further pursued to gain a deeper understanding of the pathogenesis as well as potential regimens against fibrosis.

\section{Funding}

Evangelos Karatzas is a PhD candidate at the National and Kapodistrian University of Athens. His doctoral thesis is funded by an IKY scholarship under the Action "Strengthening Human Resources, Education and Lifelong Learning", 2014-2020, co-funded by the European Social Fund (ESF) and the Greek State (MIS 5000432). Andrea Kakouri is a PhD candidate funded by the European Commission Research Executive Agency Grant BIORISE (No. 669026), under the Spreading Excellence, Widening Participation, Science with and for Society Framework. George M. Spyrou holds the Bioinformatics ERA Chair Position funded by the European Commission Research Executive Agency (REA) Grant BIORISE (Num. 669026), under the Spreading Excellence, Widening Participation, Science with and for Society Framework. 


\section{Conflict of Interest: none declared}

\section{References}

Abbaszadeh, S., et al. Memantine, an NMDA receptor antagonist, attenuates cardiac remodeling, lipid peroxidation and neutrophil recruitment in heart failure: A cardioprotective agent? Biomedicine $\mathcal{E}$ Pharmacotherapy 2018;108:1237-1243.

Argraves, W.S., et al. Fibulin is an extracellular matrix and plasma glycoprotein with repeated domain structure. The Journal of cell biology 1990;111(6):3155-3164.

Bajusz, D., Rácz, A. and Héberger, K. Why is Tanimoto index an appropriate choice for fingerprint-based similarity calculations? Journal of cheminformatics 2015;7(1):20.

Baybutt, R.C., et al. Effects on cytokines and histology by treatment with the ACE inhibitor captopril and the antioxidant retinoic acid in the monocrotaline model of experimentally induced lung fibrosis. Current pharmaceutical design 2007;13(13):1327-1333.

Bodenhofer, U., Kothmeier, A. and Hochreiter, S. APCluster: an R package for affinity propagation clustering. Bioinformatics 2011;27(17):2463-2464.

Bonner, J.C. Regulation of PDGF and its receptors in fibrotic diseases. Cytokine $\mathcal{E}$ growth factor reviews 2004;15(4):255-273.

Borkham - Kamphorst, E., Drews, F. and Weiskirchen, R. Induction of lipocalin - 2 expression in acute and chronic experimental liver injury moderated by pro - inflammatory cytokines interleukin - $1 \beta$ through nuclear factor - $\kappa \mathrm{B}$ activation. Liver International 2011;31(5):656-665.

Brown, A.S. and Patel, C.J. A standard database for drug repositioning. Scientific data 2017;4:170029.

Campillos, M., et al. Drug target identification using side-effect similarity. Science 2008;321(5886):263-266.

Carlile, G.W., et al. Ibuprofen rescues mutant cystic fibrosis transmembrane conductance regulator trafficking. Journal of Cystic Fibrosis 2015;14(1):16-25.

Chávez, E., et al. Antifibrotic and fibrolytic properties of celecoxib in liver damage induced by carbon tetrachloride in the rat. Liver International 2010;30(7):969-978.

Chen, L., et al. Differential regulation of extracellular matrix and soluble fibulin-1 levels by TGF- $\beta 1$ in airway smooth muscle cells. PLoS one 2013;8(6).

Chen, Y., Lun, A.T. and Smyth, G.K. From reads to genes to pathways: differential expression analysis of RNA-Seq experiments using Rsubread and the edgeR quasi-likelihood pipeline. F1000Research 2016;5.

Coates, A.L., et al. The effect of digoxin on exercise capacity and exercising cardiac function in cystic fibrosis. Chest 1982;82(5):543-547.

Cohen, E.P., et al. Captopril preserves function and ultrastructure in experimental radiation nephropathy. Laboratory investigation; a journal of technical methods and pathology 1996;75(3):349-360.

Cotto, K.C., et al. DGIdb 3.0: a redesign and expansion of the drug-gene interaction database. Nucleic acids research 2018;46(D1):D1068-D1073.

DeSa, J.V. LXXXVII Submucous Fibrosis of the Palate and Cheek. Annals of Otology, Rhinology E Laryngology 1957;66(4):11431159.

Duan, Q., et al. L1000CDS 2: LINCS L1000 characteristic direction signatures search engine. NPJ systems biology and applications 2016;2:16015.

Edgar, R., Domrachev, M. and Lash, A.E. Gene Expression Omnibus: NCBI gene expression and hybridization array data repository. Nucleic acids research 2002;30(1):207-210.

Flo, T.H., et al. Lipocalin 2 mediates an innate immune response to bacterial infection by sequestrating iron. Nature 2004;432(7019):917-921.

Ftahy, M.M., et al. Antifibrotic potential of a selective COX-2 inhibitor (celecoxib) on liver fibrosis in rats. Comparative Clinical Pathology 2013;22(3):425-430.

Gao, J.-H., et al. Celecoxib ameliorates portal hypertension of the cirrhotic rats through the dual inhibitory effects on the intrahepatic fibrosis and angiogenesis. PloS one 2013;8(7).

Ge, Q., et al. Fibulin1C peptide induces cell attachment and extracellular matrix deposition in lung fibroblasts. Scientific reports 2015;5:9496.

Ghazi - Khansari, M., et al. Antifibrotic effect of captopril and enalapril on paraquat - induced lung fibrosis in rats. Journal of Applied Toxicology: An International Journal 2007;27(4):342-349. 
Hallberg, P., et al. Digoxin and mortality in atrial fibrillation: a prospective cohort study. European journal of clinical pharmacology 2007;63(10):959-971.

Haller, S.T., et al. Monoclonal antibody against marinobufagenin reverses cardiac fibrosis in rats with chronic renal failure. American journal of hypertension 2012;25(6):690-696.

Hansen, M.L., et al. Aortic valve stenosis and atrial fibrillation influence plasma fibulin-1 levels in patients treated with coronary bypass surgery. Cardiology 2013;126(3):202-206.

Harris, T.R., et al. Celecoxib Does Not Protect against Fibrosis and Inflammation in a Carbon Tetrachloride-Induced Model of Liver Injury. Molecular pharmacology 2018;94(2):834-841.

Herrera, J., Henke, C.A. and Bitterman, P.B. Extracellular matrix as a driver of progressive fibrosis. The Journal of clinical investigation 2018;128(1):45-53.

Hui, A.Y., et al. Effect of celecoxib on experimental liver fibrosis in rat. Liver International 2006;26(1):125-136.

Hutchinson, J., et al. Global incidence and mortality of idiopathic pulmonary fibrosis: a systematic review. European Respiratory Journal 2015;46(3):795-806.

Jaffar, J., et al. The Serum Level Of Fibulin-1 Is Elevated In Idiopathic Pulmonary Fibrosis. In, C103. Pathogenesis, Biomarkers, and Risk Factors for Interstitial Lung Disease: from Bench to Bedside. American Thoracic Society; 2012. p. A5173-A5173.

Jaffar, J., et al. Fibulin-1 predicts disease progression in patients with idiopathic pulmonary fibrosis. Chest 2014;146(4):10551063.

Jalil, J.E., et al. Coronary Vascular Remodeling and Myocardial Fibrosis in the Rat with Renovascular Hypertension Response to Captopril. American journal of hypertension 1991;4(1_Pt_1):51-55.

Jiang, X. and Hu, J. Drug treatment of oral submucous fibrosis: a review of the literature. Journal of oral and maxillofacial surgery 2009;67(7):1510-1515.

Kanehisa, M., et al. KEGG: new perspectives on genomes, pathways, diseases and drugs. Nucleic acids research 2017;45(D1):D353-D361.

Karaman, M.W., et al. A quantitative analysis of kinase inhibitor selectivity. Nature biotechnology 2008;26(1):127-132.

Karatzas, E., et al. Exploring Fibrotic Disease Networks to Identify Common Molecular Mechanisms with IPF. In, 2019 IEEE 19th International Conference on Bioinformatics and Bioengineering (BIBE). IEEE; 2019. p. 72-77.

Karatzas, E., et al. A Web Tool for Ranking Candidate Drugs Against a Selected Disease Based on a Combination of Functional and Structural Criteria. Computational and structural biotechnology journal 2019;17:939-945.

Karatzas, E., et al. PathWalks: Identifying pathway communities using a disease-related map of integrated information. Bioinformatics (Oxford, England) 2020.

Karatzas, E., et al. PathWalks: Identifying pathway communities using a disease-related map of integrated information. BioRxiv 2020.

Karatzas, E., et al. ChemBioServer 2.0: An advanced web server for filtering, clustering and networking of chemical compounds facilitating both drug discovery and repurposing. Bioinformatics (Oxford, England) 2020.

Kim, J.-W., et al. Increased urinary lipocalin-2 reflects matrix metalloproteinase-9 activity in chronic hepatitis $C$ with hepatic fibrosis. The Tohoku journal of experimental medicine 2010;222(4):319-327.

Kim, S., et al. PubChem substance and compound databases. Nucleic acids research 2016;44(D1):D1202-D1213.

Kjeldsen, L., et al. Isolation and primary structure of NGAL, a novel protein associated with human neutrophil gelatinase. Journal of Biological Chemistry 1993;268(14):10425-10432.

Konstan, M.W., et al. Effect of high-dose ibuprofen in patients with cystic fibrosis. New England Journal of Medicine 1995;332(13):848-854.

Konstan, M.W., et al. Clinical use of ibuprofen is associated with slower FEV1 decline in children with cystic fibrosis. American journal of respiratory and critical care medicine 2007;176(11):1084-1089.

Lai, D., et al. Clinical evaluation of different treatment methods for oral submucous fibrosis. A 10 - year experience with 150 cases. Journal of oral pathology \& medicine 1995;24(9):402-406.

Lands, L.C., et al. High-dose ibuprofen in cystic fibrosis: Canadian safety and effectiveness trial. The Journal of pediatrics 2007;151(3):249-254.

Lau, J.Y., et al. Fibulin-1 is increased in asthma-a novel mediator of airway remodeling? PLoS One 2010;5(10).

$\mathrm{Li}, \mathrm{X}$. and Tang, J. Clinical treatment observation of tea pigment for oral submucous fibrosis. Hua xi kou qiang yi xue za zhi= Huaxi kouqiang yixue zazhi= West China journal of stomatology 1998;16(1):50-52.

Li, Y., et al. NMDA receptor antagonist attenuates bleomycin-induced acute lung injury. PLoS One 2015;10(5).

Lindroos, P.M., et al. Regulation of PDGFR- $\alpha$ in rat pulmonary myofibroblasts by staurosporine. American Journal of Physiology-Lung Cellular and Molecular Physiology 2001;280(2):L354-L362. 
Lindstrom, D.R., et al. Ibuprofen therapy and nasal polyposis in cystic fibrosis patients. Journal of Otolaryngology 2007;36(5). Lipinski, C.A., et al. Experimental and computational approaches to estimate solubility and permeability in drug discovery and development settings. Advanced drug delivery reviews 1997;23(1-3):3-25.

Liu, G., et al. Fibulin-1 regulates the pathogenesis of tissue remodeling in respiratory diseases. JCI insight 2016;1(9).

Liu, H., Wei, W. and Li, X. Celecoxib exacerbates hepatic fibrosis and induces hepatocellular necrosis in rats treated with porcine serum. Prostaglandins \& other lipid mediators 2009;88(3-4):63-67.

Lussier, Y.A. and Chen, J.L. The emergence of genome-based drug repositioning. Science translational medicine 2011;3(96):96ps35-96ps35.

Milner, K.L., et al. Adipocyte fatty acid binding protein levels relate to inflammation and fibrosis in nonalcoholic fatty liver disease. Hepatology 2009;49(6):1926-1934.

Mitani, H., et al. Alterations of hairless mouse skin exposed to chronic UV irradiation and its prevention by hydrocortisone. Photochemistry and photobiology 1999;69(1):41-46.

Montojo, J., et al. GeneMANIA Cytoscape plugin: fast gene function predictions on the desktop. Bioinformatics 2010;26(22):2927-2928.

Moss, A.J., Dooley, R.R. and Mickey, M.R. Cystic fibrosis complicated by heart failure. Western Journal of Medicine 1975;122(6):471.

Mostafavi, S., et al. GeneMANIA: a real-time multiple association network integration algorithm for predicting gene function. Genome biology 2008;9(1):S4.

Nakagawa, S., et al. Molecular markers of tubulointerstitial fibrosis and tubular cell damage in patients with chronic kidney disease. PLoS One 2015;10(8).

Okada, M., et al. Effects of captopril and telmisartan on matrix metalloproteinase-2 and-9 expressions and development of left ventricular fibrosis induced by isoprenaline in rats. Biological and Pharmaceutical Bulletin 2010;33(9):1517-1521.

Paik, Y.-H., et al. Celecoxib induces hepatic stellate cell apoptosis through inhibition of Akt activation and suppresses hepatic fibrosis in rats. Gut 2009;58(11):1517-1527.

Parapuram, S.K., et al. Loss of PTEN expression by dermal fibroblasts causes skin fibrosis. Journal of investigative dermatology 2011;131(10):1996-2003.

Peckham, D., et al. Effect of oral digoxin, topical ouabain and salbutamol on transepithelial nasal potential difference in patients with cystic fibrosis. Clinical Science 1995;89(3):277-284.

Powis, G., et al. Wortmannin, a potent and selective inhibitor of phosphatidylinositol-3-kinase. Cancer research 1994;54(9):2419-2423.

Raghu, G., et al. Diagnosis of idiopathic pulmonary fibrosis. An official ATS/ERS/JRS/ALAT clinical practice guideline. American journal of respiratory and critical care medicine 2018;198(5):e44-e68.

Rajendran, R., Rani, V. and Shaikh, S. Pentoxifylline therapy: a new adjunct in the treatment of oral submucous fibrosis. Indian Journal of dental research 2006;17(4):190.

Ramos, S.G., et al. Captopril reduces collagen and mast cell and eosinophil accumulation in pig serum - induced rat liver fibrosis. Pathology international 1994;44(9):655-661.

Ritchie, M.E., et al. limma powers differential expression analyses for RNA-sequencing and microarray studies. Nucleic acids research 2015;43(7):e47-e47.

Robinson, M.D., McCarthy, D.J. and Smyth, G.K. edgeR: a Bioconductor package for differential expression analysis of digital gene expression data. Bioinformatics 2010;26(1):139-140.

Rosenbloom, J., et al. Human fibrotic diseases: current challenges in fibrosis research. In, Fibrosis. Springer; 2017. p. 1-23.

Shannon, P., et al. Cytoscape: a software environment for integrated models of biomolecular interaction networks. Genome research 2003;13(11):2498-2504.

Sharma, J., et al. Clinical experience with the use of peripheral vasodilator in oral disorders. International journal of oral and maxillofacial surgery 1987;16(6):695-699.

Shi-Wen, X., et al. Endothelin-1 promotes myofibroblast induction through the ETA receptor via a rac/phosphoinositide 3kinase/Akt-dependent pathway and is essential for the enhanced contractile phenotype of fibrotic fibroblasts. Molecular biology of the cell 2004;15(6):2707-2719.

Shi-Wen, X., et al. Rac inhibition reverses the phenotype of fibrotic fibroblasts. PLoS One 2009;4(10).

Singh, M., et al. Efficacy of hydrocortisone acetate/hyaluronidase vs triamcinolone acetonide/hyaluronidase in the treatment of oral submucous fibrosis. Indian Journal of Medical Research 2010;131(5):665.

Subramanian, A., et al. A next generation connectivity map: L1000 platform and the first 1,000,000 profiles. Cell 2017;171(6):1437-1452. e1417. 
Takahashi, T., et al. A possible contribution of lipocalin - 2 to the development of dermal fibrosis, pulmonary vascular involvement and renal dysfunction in systemic sclerosis. British Journal of Dermatology 2015;173(3):681-689.

Tanabe, T., et al. IgG4-associated multifocal systemic fibrosis complicating sclerosing sialadenitis, hypophysitis, and retroperitoneal fibrosis, but lacking pancreatic involvement. Internal medicine 2006;45(21):1243-1247.

Tanramluk, D., et al. On the origins of enzyme inhibitor selectivity and promiscuity: a case study of protein kinase binding to staurosporine. Chemical biology \& drug design 2009;74(1):16-24.

Tepper, R.S., et al. Lower respiratory illness in infants and young children with cystic fibrosis. Pediatric pulmonology 1997;24(1):48-51.

Theocharis, A.D., et al. Extracellular matrix structure. Advanced drug delivery reviews 2016;97:4-27.

Tuncer, I., et al. Anti-fibrogenic effects of captopril and candesartan cilexetil on the hepatic fibrosis development in rat: the effect of AT1-R blocker on the hepatic fibrosis. Experimental and toxicologic pathology 2003;55(2-3):159-166.

Ursu, O., et al. DrugCentral 2018: an update. Nucleic acids research 2019;47(D1):D963-D970.

Veber, D.F., et al. Molecular properties that influence the oral bioavailability of drug candidates. Journal of medicinal chemistry 2002;45(12):2615-2623.

Verrecchia, F. and Mauviel, A. Transforming growth factor- $\beta$ and fibrosis. World journal of gastroenterology: WJG 2007;13(22):3056.

Vidt, D.G., Bravo, E.L. and Fouad, F.M. Captopril. New England Journal of Medicine 1982;306(4):214-219.

Wang, Y., et al. Effects of cannabinoid receptor type 2 on endogenous myocardial regeneration by activating cardiac progenitor cells in mouse infarcted heart. Science China Life Sciences 2014;57(2):201-208.

Warde-Farley, D., et al. The GeneMANIA prediction server: biological network integration for gene prioritization and predicting gene function. Nucleic acids research 2010;38(suppl_2):W214-W220.

Wen, S.L., et al. Celecoxib attenuates hepatic cirrhosis through inhibition of epithelial - to - mesenchymal transition of hepatocytes. Journal of gastroenterology and hepatology 2014;29(11):1932-1942.

Wengrower, D., et al. Prevention of fibrosis in experimental colitis by captopril: the role of TGF- $\beta 1$. Inflammatory bowel diseases 2004;10(5):536-545.

Wernig, G., et al. Unifying mechanism for different fibrotic diseases. Proceedings of the National Academy of Sciences 2017;114(18):4757-4762.

Wishart, D.S., et al. DrugBank 5.0: a major update to the DrugBank database for 2018. Nucleic acids research 2018;46(D1):D1074-D1082.

Woodruff, H., et al. Similarity measures for the classification of binary infrared data. Analytical Chemistry 1975;47(12):20272030.

Wynn, T.A. Common and unique mechanisms regulate fibrosis in various fibroproliferative diseases. The Journal of clinical investigation 2007;117(3):524-529.

$\mathrm{Xu}, \mathrm{M}$., et al. Effects of curcumin in treatment of experimental pulmonary fibrosis: a comparison with hydrocortisone. Journal of ethnopharmacology 2007;112(2):292-299.

Yu, J., et al. The anti - inflammatory effect of celecoxib does not prevent liver fibrosis in bile duct - ligated rats. Liver International 2009;29(1):25-36.

Zachariæ, L. and Zachariae, F. Hydrocortisone Acetate in the Local Treatment of Fibroses. Acta Rheumatologica Scandinavica 1957;3(1-4):76-81.

Zhang, C., et al. Celecoxib prevents pressure overload - induced cardiac hypertrophy and dysfunction by inhibiting inflammation, apoptosis and oxidative stress. Journal of cellular and molecular medicine 2016;20(1):116-127.

Zhang, X., et al. Zinc attenuates tubulointerstitial fibrosis in diabetic nephropathy via inhibition of HIF through PI-3K signaling. Biological trace element research 2016;173(2):372-383.

Zhi, H., et al. Effects of direct Renin inhibition on myocardial fibrosis and cardiac fibroblast function. PloS one 2013;8(12).

Zuberi, K., et al. GeneMANIA prediction server 2013 update. Nucleic acids research 2013;41(W1):W115-W122. 